Mon. Not. R. Astron. Soc. 000, 1-17 (0000) Printed 10 December $2021 \quad$ (MN IaT $\mathrm{E}_{\mathrm{E}} \mathrm{X}$ style file v2.2)

\title{
The Dark Matter Halos of HI Selected Galaxies
}

\author{
Saili Dutta ${ }^{1 \star}$, Nishikanta Khandai ${ }^{1} \dagger$, Sandeep Rana ${ }^{1} \ddagger$ \\ ${ }^{1}$ School of Physical Sciences, National Institute of Science Education and Research, HBNI, Jatni 752050, India
}

10 December 2021

\begin{abstract}
We present the neutral hydrogen mass $\left(M_{\mathrm{HI}}\right)$ function (HIMF) and velocity width $\left(w_{50}\right)$ function (HIWF) based on a sample of 7857 galaxies from the $40 \%$ data release of the ALFALFA survey ( $\alpha .40)$. The low mass (velocity width) end of the HIMF (HIWF) is dominated by the blue population of galaxies whereas the red population dominates the HIMF (HIWF) at the high mass (velocity width) end. We use a deconvolution method to estimate the HI rotational velocity $\left(V_{\text {rot }}\right)$ functions (HIVF) from the HIWF for the total, red, and blue samples. The HIWF and HIVF for the red and blue samples are well separated at the knee of the function compared to their HIMFs. We then use recent stacking results from the ALFALFA survey to constrain the halo mass $\left(M_{\mathrm{h}}\right)$ function of HI-selected galaxies. This allows us to obtain various scaling relations between $M_{\mathrm{HI}}-w_{50}-V_{\text {rot }}-M_{\mathrm{h}}$, which we present. The $M_{\mathrm{HI}}-M_{\mathrm{h}}$ relation has a steep slope $\sim 2.10$ at small masses and flattens to $\sim 0.34$ at masses larger than a transition halo mass, $\log _{10}\left(M_{\mathrm{ht}} h_{70}^{2} / M_{\odot}\right)=10.62$. Our scaling relation is robust and consistent with a volume-limited sample of $\alpha .40$. The $M_{\mathrm{HI}}-M_{\mathrm{h}}$ relation is qualitatively similar to the $M_{\text {star }}-M_{\mathrm{h}}$ relation but the transition halo mass is smaller by $\sim 1.4$ dex compared to that of the $M_{\mathrm{star}}-M_{\mathrm{h}}$ relation. Our results suggest that baryonic processes like heating and feedback in larger mass halos suppress HI gas on a shorter time scale compared to star-formation.
\end{abstract}

Key words: galaxies: formation, evolution, luminosity function, mass function radio lines: galaxies - surveys - cosmology: dark matter

\section{INTRODUCTION}

Over the past few decades a number of surveys using ground based and space based telescopes, have mapped different parts of the Universe, both in terms of survey area and depth, covering the entire range of the electromagnetic spectrum. These surveys have shed light on how galaxies have formed and evolved across cosmic time in cosmologically significant volumes (Madau \& Dickinson 2014; Casey, Narayanan \& Cooray 2014). Of particular interest is to understand the relative abundance of various components of galaxies, e.g. stellar populations, gas - both cold (e.g. molecular and atomic hydrogen) and hot (as seen in Xray observations of galaxy clusters) - dust, metals and also supernovae and supermassive blackholes - responsible for self-regulating the growth of galaxies through feedback processes. Targeted observations are able to capture some of

\footnotetext{
* E-mail: sailidutta@niser.ac.in (SD)

$\dagger$ E-mail: nkhandai@niser.ac.in (NK)

‡ E-mail: sandeepranaiiser@gmail.com (SR)
}

these individual properties of galaxies and allow us to look for correlations between them. These scaling relations give us some insight into galaxy formation and are also used as inputs for subgrid prescriptions in theoretical models of galaxy formation, e.g. semi-analytical models (Somerville \& Davé 2015) and cosmological hydrodynamical simulations (Naab \& Ostriker 2017).

The common ingredient in both approaches is an $N$-body code which determines how initial density perturbations evolve to form large scale structures under gravitational instability, assuming a cosmological model which is now observationally constrained to better than 10\% (Planck Collaboration, Ade et al. 2016). In the former, the entire merger tree of halos is used as a skeleton on which semianalytical recipes for galaxy formation are assigned and model parameters are tuned to reproduce only certain observations. In this work (section 5) we will use one such galaxy catalog (Knebe et al. 2018) which is calibrated to reproduce the stellar mass function of galaxies at $z=0$. Cosmological hydrodynamical simulations, on the other hand, attempt to self-consistently evolve baryonic processes, so as to repro- 
duce as many observational properties across cosmic time. However since these simulations are unable to resolve processes like star formation, supernovae explosions or gas accretion onto supermassive blackholes (to name a few) they resort to subgrid prescriptions which are calibrated from observations. Over the years, these simulations have refined their feedback models and are able to reproduce a number of observations, like the buildup of stellar mass and the star formation history of the Universe, stellar and quasar abundances and clustering and even scaling relations which have not been used as inputs in the simulations (Di Matteo et al. 2012; Vogelsberger et al. 2014; Schaye et al. 2015; Khandai et al. 2015; Crain et al. 2015; Feng et al. 2016; Pillepich et al. 2018; Davé et al. 2019).

It is only in recent years the attention has turned to reproducing and predicting (albeit qualitatively) the distribution of cold gas (e.g. atomic $\mathrm{HI}$ and/or molecular $\mathrm{H}_{2}$ hydrogen) in galaxies both in semi-analytical models (Kim, et al. 2017; Spinelli et al. 2020) and hydrodynamical simulations (Crain et al. 2017; Villaescusa-Navarro et al. 2018; Diemer et al. 2019; Davé et al. 2020; Stevens et al. 2021). This has been achieved in simulations by post processing simulation data after accounting for self-shielding (Rahmati et al. 2013). Observationally cold gas $\left(\mathrm{HI}+\mathrm{H}_{2}\right)$ is correlated to the star formation rate (SFR) - a relation also known as the Kennicutt-Schmidt law (Schmidt 1959, 1963; Kennicutt 1989, 1998) - and represents a reservoir of fuel for future star formation in galaxies. This relation also motivates the subgrid model (Springel \& Hernquist 2003) for star formation in cosmological hydrodynamical simulations outlined earlier. The simulated predictions of cold gas are at a very nascent stage and have to be refined to predict, not only abundances, but also clustering and bivariate (Zwaan, Briggs, \& Sprayberry 2001; Lemonias et al. 2013) or conditional mass functions of gas rich galaxies (Dutta, Khandai, \& Dey 2020; Dutta \& Khandai 2021, hereafter D20, D21 respectively).

Alternately there have been empirical and statistical approaches, like the Halo Abundance Matching (HAM) technique (Conroy \& Wechsler 2009; Behroozi, Conroy, \& Wechsler 2010) applied to HI (Khandai, et al. 2011; Padmanabhan \& Kulkarni 2017; Stiskalek et al. 2021) and HI halo models (Guo et al. 2017; Padmanabhan, Refregier, \& Amara 2017; Paul, Choudhury, \& Paranjape 2018; Obuljen et al. 2019; Paranjape, Choudhury, \& Sheth 2021; Paranjape et al. 2021) which have attempted to relate HI to dark matter halos based on the abundances and clustering of HI selected galaxies.

In terms of detections, gas observations have steadily increased over the past decade (for a compilation of results see Carilli \& Walter 2013; Rhee, et al. 2018, and references therein) but are still outnumbered by observations which target the stellar component of galaxies, e.g. optical, UV or IR surveys. This missing link, especially at redshifts $z \gtrsim 0.1$, between gas and star formation in galaxies needs to be bridged both by observations and theoretical models. However our understanding of the relation between cold gas and galaxy and halo environment is still limited and we need to observationally put as many constraints as possible. Huang, et al. (2012); Romeo (2020); Romeo, Agertz, \& Renaud (2020) used data from HI, optical and UV sur- veys and observationally constrained mutivariate HI-stellar scaling relations for HI selected galaxies.

In the first part of this work we use a catalog of $\mathrm{HI}$ selected galaxies to obtain the bivariate (HI-mass - velocity width) abundance of these galaxies. We use this result to obtain scaling relations between HI mass, velocity width and rotational velocity of these HI selected galaxies and also for different optically defined populations among them. We then use a recent stacking result of Guo et al. (2020) to define an HI selected halo mass function. This allows us to obtain a relation between HI mass and halo mass for an HI selected sample and also obtain scaling relations between halo mass and other HI properties like HI rotational velocities.

We assume a flat cosmology with $\left(\Omega_{m}, \Omega_{\Lambda}, h\right)$ $=(0.3,0.7,0.7)$, where $h$ is the dimensionless Hubble parameter related to the Hubble constant $H_{0}=$ $100 \mathrm{hm} \cdot \mathrm{s}^{-1} \mathrm{Mpc}^{-1}$. We follow the notation of Zwaan, et al. (2010) to define a normalized Hubble constant, $h_{70}=$ $h / 0.7=H_{0} /\left(70 \mathrm{~km} . \mathrm{s}^{-1} \mathrm{Mpc}^{-1}\right)$ which takes the value of 1 for our choice of $h=0.7$.

Our paper is organized as follows. In section 2 we describe our data and sample. In section 3 we describe a likelihood method used to estimate the HI velocity width function (HIWF). We outline a deconvolution method to obtain the HI velocity function (HIVF) from the HIWF in section 4 and present our results for the HIWF and HIVF for the full, red and blue samples of HI selected galaxies. In section 5 we present scaling relations between $\mathrm{HI}$ properties and between $\mathrm{HI}$ and halo properties. We discuss and summarize our results in section 6 .

\section{DATA}

The Arecibo Legacy Fast ALFA (ALFALFA) is a blind extragalactic HI survey, detecting galaxies with HI masses ranging from $10^{6}$ to $10^{11}$ solar masses out to redshift $z=0.06$. The $40 \%$ data release (Haynes, et al. 2011) of ALFALFA $(\alpha .40)$ is a catalog of 15855 galaxies, detected in the region $7^{h} 30^{m}<$ R.A. $<16^{h} 30^{m}, 4^{\circ}<$ dec. $<16^{\circ}$, and $24^{\circ}<$ dec. $<28^{\circ}$ and $22^{h}<$ R.A. $<3^{h}, 14^{\circ}<$ dec. $<16^{\circ}$, and $24^{\circ}<$ dec. $<32^{\circ}$. The $\alpha .40$ survey area is $\sim 2752 \mathrm{deg}^{2}$, which is $40 \%$ of the total targeted area. Most of the galaxies from ALFALFA have been crossmatched with Sloan Digital Sky Survey (SDSS) data release 7 (DR7) (Abazajian, et al. 2009). Along with the observational properties like angular position (right ascension (RA), declination (dec)), heliocentric velocity ( $\left.c z_{\text {helio }}\right)$, observed velocity profile width $\left(w_{50}\right)$, integrated flux $\left(S_{21}\right)$; some derived properties, e.g. HI mass $\left(M_{\mathrm{HI}}\right)$, distance $(D)$ are also tabulated in the catalog. The catalog also includes a flag which classifies the detection quality, based on the signal to noise ratio $(\mathrm{S} / \mathrm{N})$. Code 1 objects $\left(N_{\text {gal }}=11941\right)$ are the best detections with $S / N>6.5$. Detections with $S / N<6.5$ are referred to as code 2 objects $\left(N_{\text {gal }}=3100\right)$. The catalog contains some High Velocity Clouds (HVC), denoted by code 9 objects $\left(N_{\mathrm{HVC}}=814\right)$.

We consider only the code 1 objects for our analysis and restrict our sample to $c z_{\mathrm{cmb}}=15000 \mathrm{~km} \cdot \mathrm{s}^{-1}$ to avoid Radio Frequency Interference (RFI) (Martin, et al. 2010; Haynes, et al. 2011), where $z_{\mathrm{cmb}}$ is the galaxy redshift in 
the CMB reference frame. In this work we consider an area $\left(\sim 2093 \mathrm{deg}^{2}\right)$ common to both ALFALFA and SDSS (as discussed in D20), which contains 8344 HI-selected galaxies.

From the observed distribution of HI-selected galaxies in the $S_{21}-w_{50}$ plane (Haynes, et al. 2011, D20) we see that at fixed integrated flux, $S_{21}$, galaxies having narrower velocity width profiles are more likely to be detected. The sensitivity limit depends therefore on both flux and velocity width. We apply the sensitivity limit given by the $50 \%$ completeness limit (see eqs.4-5 of Haynes, et al. 2011), which reduces the sample to 7857 galaxies. Among these, 148 galaxies (referred to as dark galaxies) do not have any optical counterparts in SDSS DR7 although they belong to the DR7 footprint. We exclude these galaxies in this work as they will not affect our results (see D20). For the remaining 7709 galaxies we extract the ugriz magnitudes from SDSS (extinction corrected - our galaxy) and kcorrect (Blanton \& Roweis 2007) them to obtain rest frame magnitudes. kcorrect also estimates stellar masses which we will use later in this work. We obtain galaxy age estimates based on the Granada Flexible Stellar Population Synthesis (FSPS) models (Conroy, Gunn, \& White 2009; Ahn et al. 2014) from SDSS. We point out that while using the estimates from kcorrect we have not corrected for internal reddening due to dust, but as shown in D20 our results do not change considerably with those of Huang, et al. (2012), who have corrected for reddening by using two additional UV bands of GALEX (Galaxy Evolution Explorer).

A clear bimodality is seen in the distribution of SDSS galaxies in the color $(u-r)$ - magnitude $\left(M_{r}\right)$ plane. We use an optimal divider (Baldry, et al. 2004) to further classify our sample as red or blue galaxies. Red(blue) galaxies lie above(below) the optimal divider defined in the colormagnitude plane as

$C_{u r}^{\prime}\left(M_{r}\right)=2.06-0.244 \tanh \left[\frac{M_{r}+20.07}{1.09}\right]$

We have $N_{\text {gal }}^{\text {red }}=1290$ red galaxies and $N_{\text {gal }}^{\text {blue }}=6419$ blue galaxies in our observed sample.

In this work we restrict ourselves with the $\alpha .40$ sample rather than the recently released $100 \%$ catalog $(\alpha .100)$ (Haynes, et al. 2018; Durbala et al. 2020). We find that a number of galaxies in ALFALFA with optical counterparts as seen in the SDSS images, have been masked out due to bright foreground stars. Additional work will be required to extract the photometric properties of such galaxies which we will address in the future.

\section{ESTIMATING THE HI VELOCITY WIDTH FUNCTION}

Similar to the HI mass function (HIMF), the HI velocity width function (HIWF) can be defined as the underlying number density of galaxies with velocity widths in the range $\left[w_{50}, w_{50}+d w_{50}\right]$,

$$
\phi\left(w_{50}\right)=\frac{1}{V} \frac{d N}{d w_{50}}
$$

where, $d N$ is the total number of galaxies in volume $V$ having velocity widths within $w_{50}$ and $\left(w_{50}+d w_{50}\right)$. The HIWF is well described by a modified Schechter function (Zwaan, et al. 2010; Papastergis, et al. 2011; Moorman, et al. 2014)

$$
\begin{aligned}
\phi\left(w_{50}\right) & =\frac{d n}{d \log _{10} w_{50}} \\
& =\ln (10) \phi_{*}\left(\frac{w_{50}}{w_{*}}\right)^{\alpha} \exp \left[-\left(\frac{w_{50}}{w_{*}}\right)^{\beta}\right]
\end{aligned}
$$

where $\phi_{*}$ is the amplitude, $\alpha$ is the slope at the low velocity width end, $w_{*}$ is the characteristic velocity width, or the knee of the Schechter function, and $\beta$ modifies the exponential suppression at high velocity widths. For the rest of the paper we will quote the amplitude of the Schechter function, $\phi_{*}$, and the knee of the HIWF, $w_{*}$, in units of $\left(h_{70}^{3} \mathrm{Mpc}^{-3} \mathrm{dex}^{-1}\right)$ and $\log _{10}\left[w_{*} /\left(\mathrm{km} . \mathrm{s}^{-1}\right)\right]$ respectively. Later on we will also look at the HI velocity function (HIVF), described by a modified Schechter function whose knee, $V_{*}$, will similarly be in the same units as $w_{*}$. Finally the values of the HI mass, $M_{\mathrm{HI}}$, (and the knee of the HIMF, $\left.M_{*}\right)$ will be in units of $\log \left(M_{\mathrm{HI}} / M_{\odot}\right)+2 \log h_{70}$. Similarly, the values of halo mass, $M_{\mathrm{h}}$, and stellar mass, $M_{\text {star }}$, will be in units of $\log \left(M_{\mathrm{h}} / M_{\odot}\right)+2 \log h_{70}$ and $\log \left(M_{\text {star }} / M_{\odot}\right)+$ $2 \log h_{70}$, respectively, throughout the paper.

The step-wise maximum likelihood (SWML) (Efstathiou, Ellis, \& Peterson 1988) is used to estimate the underlying velocity width function. This model-independent method does not assume any functional form of the width function, rather it estimates a discretized (or binned) width function, $\phi\left(w_{50}^{k}\right)$. Being a maximum likelihood method, it is insensitive to the effects of local variations in galaxy densities due to clustering. For HI-selected galaxies the detection probability depends both on $M_{\mathrm{HI}}$ and $w_{50}$. We implement a two-dimensional SWML method (2DSWML) to first estimate the bivariate HI mass-velocity width function, $\phi\left(M_{\mathrm{HI}}, w_{50}\right)$ and then integrate over $M_{\mathrm{HI}}$ to obtain the HIWF. The approach is similar to Loveday (2000); Zwaan, et al. (2003); Martin, et al. (2010); Haynes, et al. (2011) and has been described in detail in D20 in the context of obtaining the HIMF. We briefly outline the method below.

To estimate the bivariate distribution function $\phi_{j k} \equiv$ $\phi\left(M_{\mathrm{HI}}^{j}, w_{50}^{k}\right)$, we divide the HI mass range and HI velocity width range into logarithmic $n_{M}$ and $n_{W}$ bins, where $j=$ $0,1, . ., n_{M}-1$ and $k=0,1, . ., n_{W}-1$. The probability of detecting a galaxy $i$ of HI mass $M_{\mathrm{HI}}^{i}$ and HI velocity width $w_{50}^{i}$ at a distance $D^{i}$ is

$p_{i}=\frac{\Sigma_{j} \Sigma_{k} V_{i j k} \phi_{j k}}{\Sigma_{j} \Sigma_{k} H_{i j k} \phi_{j k} \Delta M \Delta W}$

where $\Delta M$ and $\Delta W$ are the bin widths in the mass $M=\log _{10}\left[M_{\mathrm{HI}} / M_{\odot}\right]$ and velocity width $W=$ $\log _{10}\left[w_{50} /\left(\mathrm{km}_{\mathrm{s}}{ }^{-} 1\right)\right]$ axes respectively. $V_{i j k}$ is an occupation number and takes a value of 1 (0 otherwise) if the galaxy $i$ is binned in the ' $j k$ ' bin, $\Sigma_{i} V_{i j k}=n_{j k}$, where $n_{j k}$ is the observed number of galaxies in ' $j k$ ' bin. $H_{i j k}$ takes care of the completeness limit of the sample and represents the fractional area in the $M_{\mathrm{HI}}-w_{50}$ plane that is accessible to the $i^{t h}$ galaxy. It takes values from 0 to 1 (See Dutta, Khandai, \& Dey 2020). The joint likelihood, $\mathcal{L}=\prod_{i=1}^{N_{\text {gal }}} p_{i}$ is then maximized with respect to $\phi_{j k}$, which gives us an expression for $\phi_{j k}$

$\phi_{j k}=n_{j k}\left[\Sigma_{i} \frac{H_{i j k}}{\Sigma_{m} \Sigma_{n} H_{i m n} \phi_{m n}}\right]^{-1}$ 

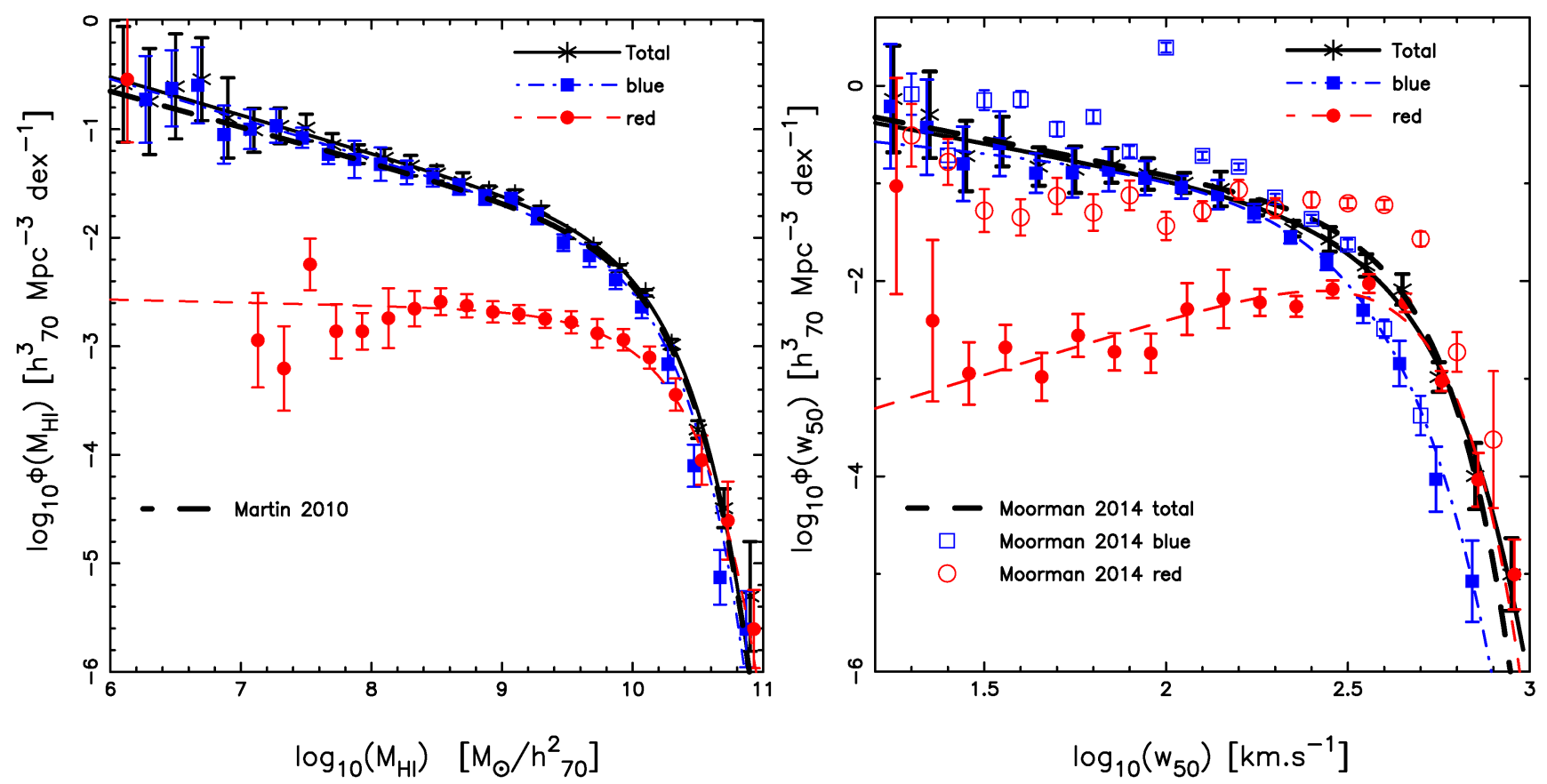

Figure 1. Our estimates of the HIMFs (HIWFs) are shown in the left (right) panel for the total (cross), blue (filled square) and red (filled circle) samples. To better illustrate our results the data for the red (blue) sample have been horizontally offset rightward (leftward) with respect to the total sample. The Schechter (modified Schechter) function fits are plotted for the total (thick solid line), red (thin dashed line) and blue (thin dot-dashed line) samples for the HIMFs (HIWFs). The thick dashed line in the left panel is the estimate of the HIMF from Martin, et al. (2010). The thick dashed line in the right panel is the estimate of the HIWF from Moorman, et al. (2014). Both of these have used the $\alpha .40$ sample. The open circles (squares) in the right panel are the unnormalized estimates of the HIWF for the red (blue) sample from Moorman, et al. (2014)

which needs to be solved iteratively. The iteration is started by setting $\phi_{j k}$ to $n_{j k} / V$ and is stopped when we achieve a minimum $1 \%$ accuracy for all $\phi_{j k}$. Finally we integrate the bivariate $\phi_{j k}$ over $M_{\mathrm{HI}}$ to obtain the HIWF:

$\phi_{k}=\sum_{j} \phi_{j k} \Delta M$

Integrating $\phi_{j k}$ over $w_{50}$, on the other hand, gives us the HIMF $\phi_{j}$. A common feature of maximum likelihood methods is that the normalization of $\phi\left(M_{\mathrm{HI}}, w_{50}\right)$ needs to be fixed separately since it gets lost in the process (see equation 4). We do this by computing the selection function and matching the observed number density to the underlying number density convolved by the selection function (Davis \& Huchra 1982; Martin, et al. 2010).

\subsection{Uncertainties on HIWF}

We consider the following four sources of error and add them in quadrature to quantify the uncertainty on the estimated HIWF, similar to Moorman, et al. (2014).

(i) Velocity width errors: Errors due to the measurement of $w_{50}$ can change the occupation of one galaxy in $M_{\mathrm{HI}}-w_{50}$ plane. To account for this we have considered 300 realizations of $w_{50}$ (Gaussian random realizations using $w_{50}$ as the mean and $\sigma_{w_{50}}$ as the variance) and estimated HIWF for each of these realizations. The distribution of $\phi_{k}$ for a fixed $k$ gives an estimate of the uncertainty on $\phi_{k}$.

(ii) Distance errors: HI mass $\left(M_{\mathrm{HI}}\right)$ depends on distance, and since we estimate the HIWF from a bivariate function of $M_{\mathrm{HI}} \& w_{50}$, the measurement errors on distance is also a source of uncertainty. We follow a similar approach as above to get the errors on $\phi_{k}$, by generating 300 random realizations.

(iii) Sample variance: We split our survey area into 26 regions of approximately equal angular area and estimate the HIWF by eliminating one region at one time (the Jackknife sample). We then estimate the Jackknife uncertainty as $\sigma_{\phi_{k}}=\frac{N-1}{N} \sum_{i=1}^{N}\left(\bar{\phi}_{k}-\phi_{k}^{i}\right)^{2}$ where $N$ is the number of Jackknife samples, $\overline{\phi_{k}}$ is the Jackknife mean and $\phi_{k}^{i}$ is the value for the $i^{\text {th }}$ Jackknife sample.

(iv) Poisson errors: The observed counts at the two ends of $\phi\left(w_{50}\right)$ are small, it is therefore important to consider Poisson errors.

\section{HI VELOCITY WIDTH FUNCTION}

In this section we present the HIMF and the HIWF for the full sample as well as for the red and blue samples. We then use a deconvolution (or inversion) method (Papastergis, et al. 2011) to estimate the HIVF from the HIWF.

In figure 1 we present our estimates of the HIMF (left panel) and the HIWF (right panel) for the total (cross), red (filled circle) and blue (filled square) samples. Error estimates on the HIMF are described in D20 and are similar to the error estimates for the HIWF. To better display our data we have horizontally offset data for the red and blue samples with respect to the total sample. The thick solid, thin dashed and thin dot-dashed lines are our Schechter (mod- 

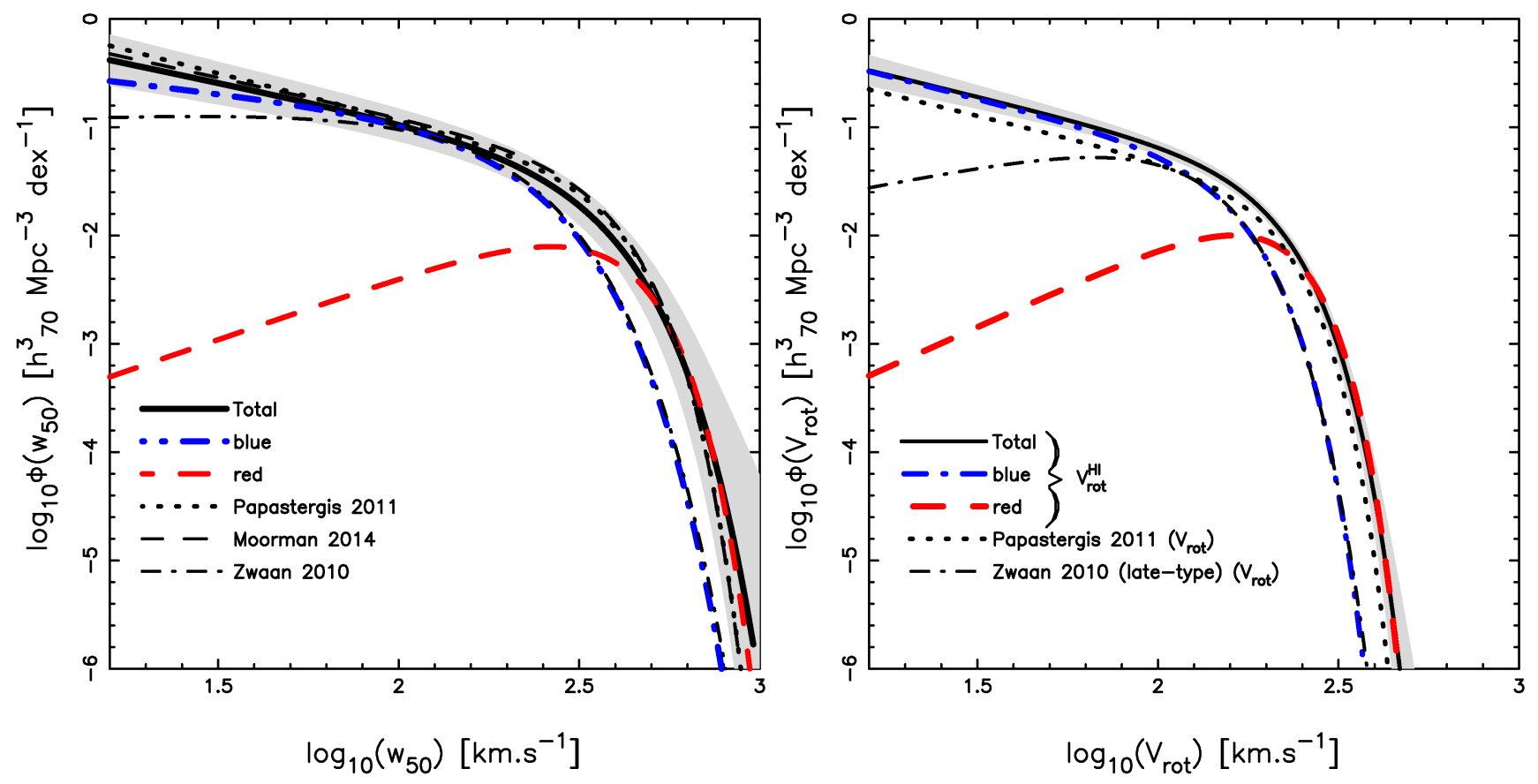

Figure 2. A comparison of our estimates with previously published results of the HIWF (HIVF) are shown in the left (right) panel. Our modified Schechter function fits and their $1 \sigma$ uncertainty (shaded region for the total sample only) are plotted for the total (thick solid line), red (thin dashed line) and blue (thin dot-dashed line) samples in both panels. The thick dotted line represents the estimates of Papastergis, et al. (2011) for both the HIWF and the HIVF. The thin dot-dashed lines are the estimates of Zwaan, et al. (2010) from the HIPASS data for the full sample (left panel, HIWF) and for late-type galaxies (right panel, HIVF). The thin dashed line is the estimate of the HIWF of Moorman, et al. (2014).

ified Schechter) function fits to the HIMF (HIWF) for the total, red and blue samples. We compare our results for the HIMF (HIWF) with Martin, et al. (2010) (Moorman, et al. 2014) (thick dashed line). We find that our estimates compare well with these authors for the total sample. Moorman, et al. (2014) also estimated the HIWF for the red (open circles) and blue (open square) samples but they are most likely unnormalized since they do not add up to give the HIWF for the total sample. The overall shape of the red sample compares well with Moorman, et al. (2014); however, since the binning is different, it is difficult to make a point by point comparison. Moorman, et al. (2014) have an outlier at $w_{50} \approx 100 \mathrm{~km} . \mathrm{s}^{-1}$. We find that it comes from a single galaxy which is extremely gas poor with $M_{\mathrm{HI}}=6.78$. We have removed this object in our analysis.

Galaxies have random inclinations, therefore we relate the observed HI profile width, $w_{50}$, to the intrinsic HI rotational velocity of the galaxy, $V_{\text {rot }}^{\mathrm{HI}}$, by

$$
w_{50}=2 V_{\mathrm{rot}}^{\mathrm{HI}} \sin i+w_{\mathrm{nr}}
$$

This is similar to the approach taken by Zwaan, et al. (2010); Papastergis, et al. (2011). Here $i$ is the inclination angle and $w_{\mathrm{nr}}$ is an additional term which captures broadening by turbulence and other non-rotational motion. We will discuss later the use of eq. 7 which is valid when $w_{\mathrm{nr}} \ll V_{\text {rot }}^{\mathrm{HI}}$.

Although it is tempting to relate $V_{\text {rot }}^{\mathrm{HI}}$ to rotational velocities, $V_{\text {rot }}$, associated with rotation curve measurements by radio synthesis observations, one needs to be careful in this regard. Verheijen (2001) classified rotation curves broadly as i. rising (R-type) ii. flat (F-type) and iii. declining (D-type) rotation curves. R-type rotation curves (Li et al.
2020) are generally associated with low-surface brightness or dwarf galaxies and the observed maximum rotational velocity, $V_{\max }$, is the last point of the rotation curve measurement. It represents a lower bound to the rotational velocity, $V_{\text {rot }}$, associated with the gravitational potential. F-type rotation curves ( $\mathrm{Li}$ et al. 2020) are the classical flat rotation curves that extend beyond the optical radius. The rotation curves initially rise, reach a maximum value $V_{\max }$, and taper off to a slightly lower, constant value, $V_{\text {flat }} \lesssim V_{\text {max }}$, at large radii. $V_{\text {flat }}$ is associated with the maximum rotational velocity, $V_{\text {rot }}$, that would be induced by the mass of the halo. Ftype curves are associated with late-type spirals and $V_{\text {rot }}^{\mathrm{HI}}$, in equation 7 , can be associated with $V_{\text {flat }}=V_{\text {rot }}=V_{\text {rot }}^{\mathrm{HI}}($ Verheijen 2001). Finally the D-type curves (Lelli et al. 2017), seen in early-type galaxies (ETGs), are characterized by an increasing rotation curve reaching to $V_{\max }$ within the stellar disk and declining thereafter beyond the optical radius to either a constant value, $V_{\text {flat }}$, or to a smaller value, $V_{\min }$. In the D-type curves, $V_{\max }>V_{\text {flat }}, V_{\min }$.

Among the small fraction of ETGs detected in HI, in the ATLAS ${ }^{3 D}$ survey (Serra et al. 2012) most have settled HI disk configurations which are rotating. However while creating dynamical mass models based on the rotation curves (Lelli, McGaugh, \& Schombert 2016; Lelli et al. 2017) one has to also consider stellar velocity dispersion and pressure support of ionized X-ray gas in such systems (Lelli et al. 2017). The corresponding $V_{\text {rot }}$ based on these models is not the same as $V_{\text {rot }}^{\mathrm{HI}}$. Pressure support is also important in dwarfs described by R-type rotation curves.

Given these considerations we will interpret $V_{\text {rot }}^{\mathrm{HI}}$ in equation 7 as the HI profile width if the HI disk were seen 


\begin{tabular}{ccccccccc}
\hline & \multicolumn{3}{c}{$\phi\left(w_{50}\right)$} & & \multicolumn{3}{c}{$\phi\left(V_{\text {rot }}^{\mathrm{HI}}\right)$} \\
\hline & $\phi_{*}$ & $w_{*}$ & $\alpha$ & $\beta$ & $\phi_{*}$ & $V_{*}$ & $\alpha$ & $\beta$ \\
\hline total & $0.023 \pm 0.008$ & $2.50 \pm 0.06$ & $-0.70 \pm 0.16$ & $2.02 \pm 0.23$ & $0.0187 \pm 0.0023$ & $2.3 \pm 0.01$ & $-0.81 \pm 0.13$ & $2.7 \pm 0.17$ \\
\hline blue & $0.046 \pm 0.014$ & $2.29 \pm 0.06$ & $-0.37 \pm 0.18$ & $1.74 \pm 0.16$ & $0.0206 \pm 0.0023$ & $2.2 \pm 0.01$ & $-0.84 \pm 0.16$ & $2.7 \pm 0.18$ \\
\hline red & $0.008 \pm 0.002$ & $2.56 \pm 0.07$ & $1.14 \pm 0.29$ & $2.51 \pm 0.45$ & $0.0102 \pm 0.0013$ & $2.31 \pm 0.01$ & $1.5 \pm 0.18$ & $2.96 \pm 0.14$ \\
\hline
\end{tabular}

Table 1. Best fit values of modified Schechter parameters for $\phi\left(w_{50}\right)$ and $\phi\left(V_{\mathrm{rot}}^{\mathrm{HI}}\right)$ for total, blue and red samples.

edge-on, corrected for non-rotational broadening. In figure 8 we see that the $M_{\mathrm{HI}}-V_{\text {rot }}^{\mathrm{HI}}$ relation in our sample is broadly consistent with the $M_{\mathrm{HI}}-V_{\text {flat }}$ measurements from the Spitzer Photometry and Accurate Rotation Curves (SPARC)(Lelli, McGaugh, \& Schombert 2016) sample.

Broadly there are two approaches in obtaining $V_{\text {rot }}^{\mathrm{HI}}$ from $w_{50}$. One can correct for inclination effects (Zwaan, et al. 2010) by estimating inclination angles from identified optical counterparts. This method however is accurate for inclination angles $i \leq 45^{\circ}$. Because of the ambiguity of interpreting $V_{\text {rot }}^{\mathrm{HI}}$ with $V_{\text {rot }}$ for all morphological types, Zwaan, et al. (2010) estimated the HI velocity function (HIVF) with the 2DSWML method only for late-type galaxies with this method, after applying a correction in the abundance for galaxies with inclination angles $i>45^{\circ}$. Alternately the rotation curve $V_{\text {rot }}(r)$, and the surface density of the HI disk, $\Sigma_{\mathrm{HI}}(r)$, can produce the observed HI flux density profile, $S_{\mathrm{HI}}(v)$, after convolving with the telescope beam response and non-rotational broadening (arising due to turbulent motions in the disk) and accounting for inclination (Gordon 1971; Schulman, Bregman, \& Roberts 1994; Paranjape et al. 2021). As a proof of concept, Paranjape et al. (2021) turned this expression (see their eq.3) around to infer halo properties from the observed $21 \mathrm{~cm}$ line profile, $S_{\mathrm{HI}}(v)$, of two galaxies NGC-99 and UGC-00094 in ALFALFA, by considering a baryonification model of halos to produce rotation curves (Paranjape, Choudhury, \& Sheth 2021) and using an observationally constrained scaling of the HI scale length to HI mass, $h_{\mathrm{HI}} \propto M_{\mathrm{HI}}^{0.5}$ (Wang et al. 2016), which determines $\Sigma_{\mathrm{HI}}(r) \propto \exp \left(-r / h_{\mathrm{HI}}\right)$. Paranjape et al. (2021) also showed that the mass weighted second moment of the rotational velocity is related to $w_{50}$ using this relation. Although there are many assumptions and parameters which lead us from halos to the HI line profile, this method provides a novel way to estimate the rotational velocity from the observed $21 \mathrm{~cm}$ spectrum $S_{\mathrm{HI}}(v)$. One can then use the 2DSWML method to estimate the rotational velocity function (mass weighted) of HI selected galaxies as was done by Zwaan, et al. (2010).

The second approach is a statistical method implemented by Papastergis, et al. (2011). We follow this approach. We assume that the HIVF is also a modified Schechter function described by equation 3 with parameters $\left(\phi_{*}, V_{*}, \alpha, \beta\right)$. We generate a realization of this model Schechter function and randomize their inclinations $(\cos i$ uniformly distributed) and then use equation 7 to obtain a realization of the HIWF. We use $w_{\mathrm{nr}}=5 \mathrm{~km} . \mathrm{s}^{-1}$ in equation 7 (Verheijen \& Sancisi 2001; Papastergis, et al. 2011), and add it linearly for galaxies with $V_{\text {rot }}>50 \mathrm{~km} . \mathrm{s}^{-1}$ and in quadrature for galaxies with smaller velocities (Papastergis, et al. 2011).
Although Stilp et al. (2013) refer to a bit higher values $\left(5-15 \mathrm{~km} . \mathrm{s}^{-1}\right)$ for $w_{n r}$, we find negligible differences in the derived HIVFs for different $w_{n r}$ values and stick with $w_{\mathrm{nr}}=5 \mathrm{~km} \cdot \mathrm{s}^{-1}$ in order to be consistent with Papastergis, et al. (2011). We point the reader to appendix A for a more detailed discussion on this issue.

The model HIWF is compared to our estimated binned HIWF (data points with errors in figure 1) and a $\chi^{2}$ is computed using the model, data and associated errors. Finally the best fit model parameters of the HIVF are obtained by minimizing the $\chi^{2}$ with the model parameters. We do this for the total, red and blue samples.

Our results are shown in figure 2 and summarized in table 1 . In figure 2 we compare our modified Schechter function fits with those obtained earlier with the HI Parkes All Sky Survey, (HIPASS, Meyer et al. 2004; Zwaan, et al. 2010) and the $\alpha .40$ sample of ALFALFA (Papastergis, et al. 2011; Moorman, et al. 2014). The thick solid, thick dashed and thick dot-dashed lines are our modified Schechter function fits for the total, red and blue samples. The grey region is the $1 \sigma$ uncertainty for the total sample. The dotted line is the result of Papastergis, et al. (2011). The thin dashed line in the left panel of figure 2 is the estimate of the HIWF by Moorman, et al. (2014). The thin dot-dashed line is the result of Zwaan, et al. (2010) for the HIWF (left panel, all galaxies) and the HIVF(right panel, late-type galaxies) in HIPASS.

As can be seen in figure 2, our result for the HIWF agrees well (within errors) with Papastergis, et al. (2011); Moorman, et al. (2014). There is a factor of $\sim 2$ discrepancy in $\phi_{*}$ between Papastergis, et al. (2011) $\left(\phi_{*}=0.011 \pm 0.002\right)$ and Moorman, et al. (2014) $\left(\phi_{*}=0.021 \pm 0.002\right)$. Our value of $\phi_{*}=0.023 \pm 0.008$ is in better agreement with Moorman, et al. (2014). However given the correlation between the parameters of the Schechter function the differences in other parameters compensate for each other and keep the overall shape within the $1 \sigma$ uncertainty. The difference between the ALFALFA (Papastergis, et al. 2011; Moorman, et al. 2014, this work) results and HIPASS (Zwaan, et al. 2010) are much starker. Although the full HIPASS sample has been taken to estimate the HIWF, the results from Zwaan, et al. (2010) seems to match only with the HIWF of blue galaxies in ALFALFA at the high velocity end, and has a shallower slope at lower velocity widths. The reason is that due to its higher sensitivity ALFALFA is able to detect larger velocity widths compared to HIPASS. As is evident from the figure, the large velocity end is dominated by red galaxies which one can associate with early-type galaxies (see figure 4), HIPASS would therefore miss this population altogether. The observed frac- 
tion of early type galaxies in HIPASS is $11 \%$ compared to $20 \%$ for red galaxies in our sample.

In the right panel of figure 2 we show our estimates of the HIVF for the total, red and blue samples. Comparison is made with the total sample of ALFALFA (Papastergis, et al. 2011) and the late-type galaxies in HIPASS (Zwaan, et al. 2010). Similar to what we saw in the HIWF, the high velocity end of the HIVF for the late type galaxies in HIPASS compares well with that of blue galaxies in our sample. This suggests that associating $V_{\text {rot }}^{\mathrm{HI}}$ with $V_{\text {rot }}$ is correct for late type galaxies. This also suggests that associating blue galaxies with late type galaxies with the age criterion given in figure 4 is reasonable. Finally it confirms our earlier argument that HIPASS galaxies sample the blue cloud more frequently as compared to ALFALFA.

For the total sample our results of the HIVF is systematically offset compared to Papastergis, et al. (2011) although our HIWF agree with each other. However Papastergis, et al. (2011) have estimated the rotational velocity,$V_{\text {rot }}$, function for HI-selected galaxies, whereas we have estimated the HI rotational velocity, $V_{\text {rot }}^{\mathrm{HI}}$, function. To estimate the rotational velocity function Papastergis, et al. (2011) estimated the HIVF for late type galaxies, based on the inversion method outlined above. For early type galaxies they used the velocity dispersion function, $\phi^{\text {early }}(\sigma)$, of early type galaxies obtained from SDSS and the 2dFGRS (Chae 2010). $\phi^{\text {early }}(\sigma)$ was then converted to $\phi^{\text {early }}\left(V_{\text {rot }}\right)$ assuming $V_{\text {rot }}=\sqrt{2} \sigma$ for an isothermal profile. Finally $\phi\left(V_{\text {rot }}\right)$ for ALFALFA galaxies was constructed by smoothly interpolating the HIVF for late-type galaxies at low velocities to $\phi^{\text {early }}\left(V_{\text {rot }}\right)$ at large velocities assuming a modified Schechter function. These results suggest that although $V_{\text {rot }}^{\mathrm{HI}}$ is not the same as $V_{\text {rot }}$ for early type galaxies, a systematic correction to $V_{\text {rot }}^{\mathrm{HI}}$ can be added to bring it closer to the 'true' rotational velocity.

We end this section with a final observation. We find that the HIVF and HIWF for red and blue galaxies are well separated beyond the knee of the velocity functions. The red population dominates the velocity function at larger velocities. The red population also dominates the HIMF at larger masses but the difference between the red and blue mass functions is much smaller compared to the velocity functions. The blue population dominates the abundances at lower masses and velocities, and the red population has a slope which is much shallower compared to the blue population at this end.

\section{SCALING RELATIONS FOR HI SELECTED GALAXIES}

In the last section we have observationally derived three distributions which describe the abundances of HI-selected galaxies. These are the HIMF, HIWF and HIVF. These abundances have been estimated based on their HI signal and the ALFALFA selection function. The next step is to relate HI properties to either galaxies or halos. In D20 and D21 we have presented the conditional HI mass functions, conditioned on optical color and/or magnitude for HI-selected galaxies. In what follows we will build a model to populate halos with HI.

The Halo Abundance Matching (HAM) technique is a powerful and elegant method to match halo properties to galaxy properties (Vale \& Ostriker 2004; Conroy \& Wechsler 2009; Behroozi, Conroy, \& Wechsler 2010; Moster, Naab, \& White 2013). It assumes that every dark matter halo above a certain mass threshold hosts one galaxy. In its simplest form it assumes that the most massive (or luminous) galaxy is hosted in the most massive halo, or a monotonic relation between galaxy mass and halo mass. To derive the stellarhalo mass relation of the galaxy one matches the spatial abundances of galaxies to that of halos.

$$
n\left(>M_{\text {star }}\right)=n\left(>M_{\mathrm{h}}\right)
$$

Here $n$ is the number density of objects above a certain mass threshold, $M_{\text {star }}$ (galaxy stellar mass) or $M_{\mathrm{h}}$ (halo mass). To further obtain the $M_{\text {star }}-M_{\mathrm{h}}$ relation one needs the stellar mass function (SMF) of galaxies (which are observationally constrained) and the halo mass function (which can be obtained from $N$-body simulations). The relation can include scatter between $M_{\text {star }}$ and $M_{\mathrm{h}}$, and has been shown to reproduce the clustering of galaxies (see Behroozi, Conroy, \& Wechsler 2010, and references therein). HAM techniques provide a starting point for parametrizing the $M_{\text {star- }}$ $M_{\mathrm{h}}$ scaling relation. It has been shown both observationally (Behroozi, Wechsler, \& Conroy 2013) and with hydrodynamical simulations (Chaves-Montero et al. 2016), that $M_{\text {peak }}$ (the maximum virial mass of the halo, or subhalo, over its accretion history) or $V_{\text {peak }}$ (the maximum $V_{\max }$ - the maximum circular velocity - of the halo, or subhalo, over its accretion history) is more tightly correlated to $M_{\text {star }}$ as compared to $M_{\mathrm{h}}$. Abundance matching $M_{\text {star }}$ to either $M_{\text {peak }}$ (or $\left.V_{\text {peak }}\right)$ better reproduces observed clustering data in mock catalogs. However we will show in a forthcoming paper, using the $M_{\mathrm{HI}}-M_{\mathrm{h}}$ relation (as opposed to $M_{\mathrm{HI}}-M_{\text {peak }}$ ) better reproduces HI clustering. This relation can be refined further and include redshift evolution and uncertainties so that it reproduces observed galaxy abundances and clustering for different galaxy types (Behroozi, Wechsler, \& Conroy 2013; Behroozi et al. 2019) across cosmic time.

HAM techniques have also been used to obtain the $M_{\mathrm{HI}}-M_{\mathrm{h}}$ relation (Khandai, et al. 2011; Padmanabhan \& Kulkarni 2017). However the basic assumption in HAM - a monotonic relation between halo mass and the galaxy property - may not hold if we consider the galaxy property to be the HI mass. Unlike the stellar mass the HI content of galaxies is very sensitive to its local environment and may decrease with increasing halo mass. In massive halos, e.g. clusters and groups, the virial temperature is large and feedback processes, e.g. from supermassive blackholes keep the gas ionized. It is therefore less likely, on average, to have a considerable amount of $\mathrm{HI}$ associated with such massive systems. These arguments are observationally supported. We find that in the sample and survey volume considered here, $11 \%(38 \%)$ of detections in $\mathrm{HI}$ are in the red (blue) cloud (D20). The luminous red galaxies dominate the high mass end of the galaxy SMF and at $M_{\text {star }}=11.3$ the number of red galaxies is $\sim 10 \times$ larger than the blue galaxies (Baldry, et al. 2012). These galaxies are mostly centrals (Drory, et al. 2009) and would be hosted in halos of mass $M_{\mathrm{h}} \sim 14$ (Behroozi et al. 2019) with virial temperatures $T_{\text {vir }} \sim 10^{7} \mathrm{~K}$. It would be very rare to see large amounts of $\mathrm{HI}$ in such systems. Direct and more sensitive observations targeting massive galaxies, the GALEX Arecibo SDSS 

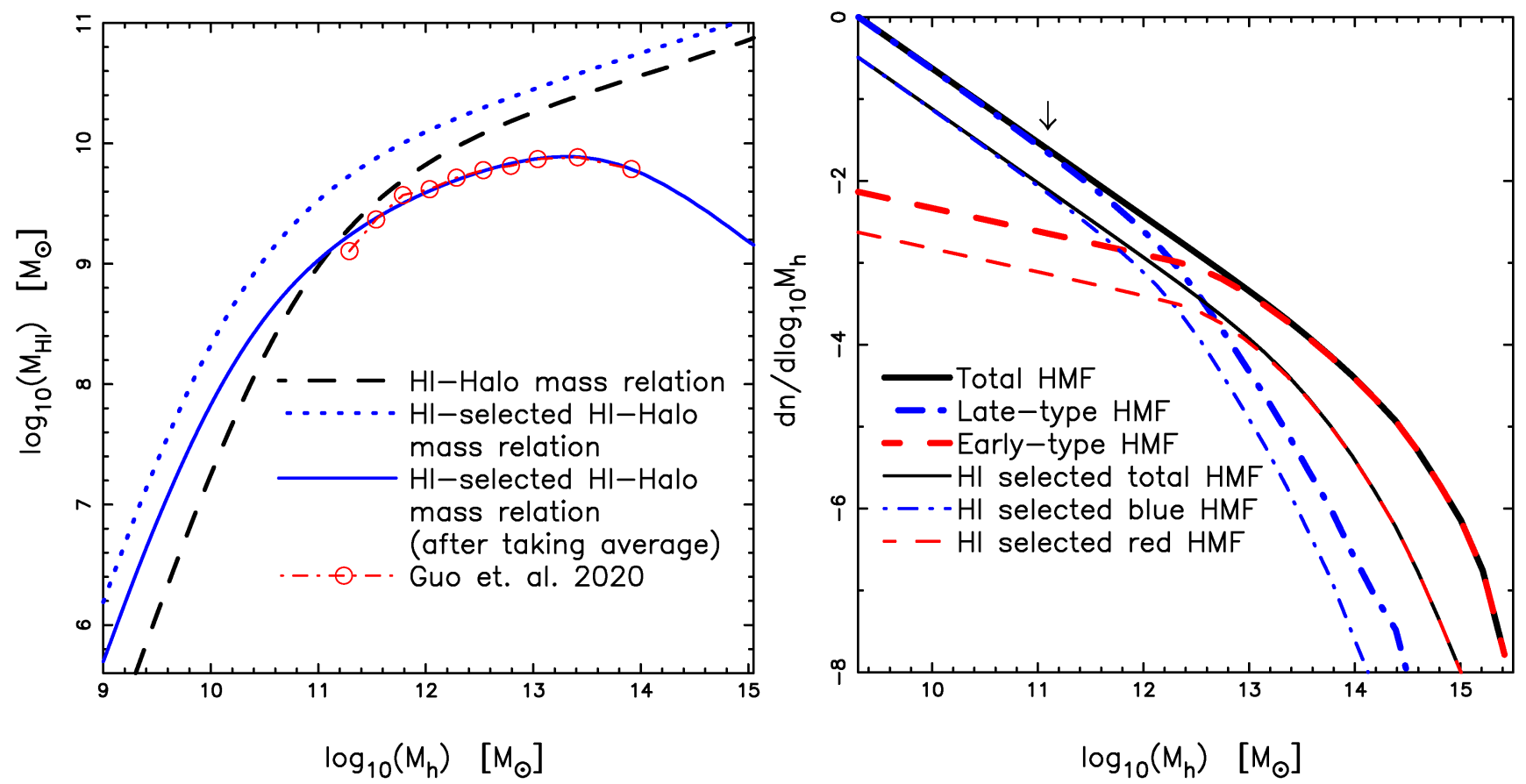

Figure 3. Left panel: Average HI mass - halo mass relation from Guo et al. (2020) is shown as open circles. The dashed line is the $M_{\mathrm{HI}}-M_{\mathrm{h}}$ scaling relation obtained by abundance matching our HIMF to the HMF. The dotted line is the $M_{\mathrm{HI}}-M_{\mathrm{h}}$ relation obtained by abundance matching our HIMF to the HI-selected HMF (see equations 9,10 and discussion). The solid line is the mean $\left\langle M_{\mathrm{HI}}\right\rangle-M_{\mathrm{h}}$ relation that is obtained by averaging the dotted line over all the halos at any given mass $\left[M_{\mathrm{h}}, M_{\mathrm{h}}+d M_{\mathrm{h}}\right]$. The HI-selected HMF has been defined so that the solid line matches the observed points of Guo et al. (2020). Right panel: The HMF (HI-selected HMF) is shown as the solid thick (thin) line. The arrow at $M_{\mathrm{h}} \simeq 11$ is the resolution of the simulation catalog. For masses below that we have linearly extrapolated. The thick dashed (dot-dashed) line represents the HMF hosting early (late) type galaxies. (See figure 4 and corresponding text in section 5 for the definition of early (late) type galaxies. The corresponding HI-selected HMF are shown in thin lines.

Survey (GASS) (Catinella, et al. 2013), and massive ETGs, the ATLAS ${ }^{3 \mathrm{D}}$ survey (Serra et al. 2012), confirm these arguments. They find that massive galaxies are dominated by non-detections in $\mathrm{HI}$ and the limiting $\mathrm{HI}$ masses (upper bound based on survey sensitivity) is well below the knee of the HIMF $\left(M_{*}=9.96\right)$. We finally note that the high mass end of the HIMF is dominated by luminous red galaxies which represent a small fraction of all luminous red galaxies. As mentioned earlier luminous red galaxies dominate the SMF (hence the halo mass function) over their blue counterparts by at least a factor of 10 . It is not surprising,therefore, to see that the HIMF is dominated by red galaxies since a small fraction of gas rich red galaxies is all that is needed to boost their abundances over that of their blue counterparts.

We have argued that HAM cannot be applied to obtain the $M_{\mathrm{HI}}-M_{\mathrm{h}}$ relation since we do not expect a monotonic correlation between $\mathrm{HI}$ masses and stellar or halo masses. In what follows, given the tight monotonic relation between stellar and halo masses, we will use the halo and stellar masses as proxies for each other. Although a stellar mass selected sample should not have a monotonic relation with $\mathrm{HI}$ mass, we can turn this around and ask if an HI-selected sample like ours has a monotonic relation with stellar mass. This is indeed true and has been seen both in ALFALFA (Huang, et al. 2012, D20) and the HI Parkes All-Sky Survey Catalog (HICAT) (Maddox, et al. 2015). One can therefore expect that gas rich galaxies will, on average, be found in more massive halos as compared to gas poor halos. A sub- sample of all halos should host $\mathrm{HI}$ in such a manner that the relation between $M_{\mathrm{HI}}$ and $M_{\mathrm{h}}$ is monotonic.

Recently Guo et al. (2020) used ALFALFA with an optical group catalog from SDSS to estimate the mean HI mass - halo mass, $\left\langle M_{\mathrm{HI}}\right\rangle-M_{\mathrm{h}}$, relation by stacking $\mathrm{HI}$ on the optically selected group catalog. The HI was stacked for the full group and separately for centrals in the catalog, the difference between the two therefore represents the contribution of total HI mass in satellites in the group. Due to confusion, stacking of centrals is contaminated by nearby satellites within the group. Therefore the $\left\langle M_{\mathrm{HI}}^{\mathrm{cen}}\right\rangle$ for centrals are upper limits, the $\left\langle M_{\mathrm{HI}}^{\mathrm{sat}, \text { tot }}\right\rangle$ for satellites are lower limits and the $\left\langle M_{\mathrm{HI}}^{\text {group }}\right\rangle$ is the average stacked mass in groups. The stacking procedure was carried out as a function of group or halo mass. In the left panel of figure 3 the result of Guo et al. (2020) $\left\langle M_{\mathrm{HI}}\right\rangle-M_{\mathrm{h}}$ is shown for centrals (open circles).

At this stage it is important to clarify how we associate $\mathrm{HI}$ in galaxies and halos. Galaxies can be in all kinds of environments. They can be isolated field galaxies, central galaxies in groups and clusters or satellite galaxies in such systems. These are distinct objects in observations. The halo on the other hand can be defined as a concentration of mass within a radius such that it is virialized inside this radius (central halo). Simulations show that halos have substructure and sub-substructure (and so on) and these objects (satellite halo) are not only self-bound but bound to the halo. In what follows we will refer to a halo as either a central halo or satellite halo and will not distinguish between them. Most of the mass inside the radius of a halo is associ- 

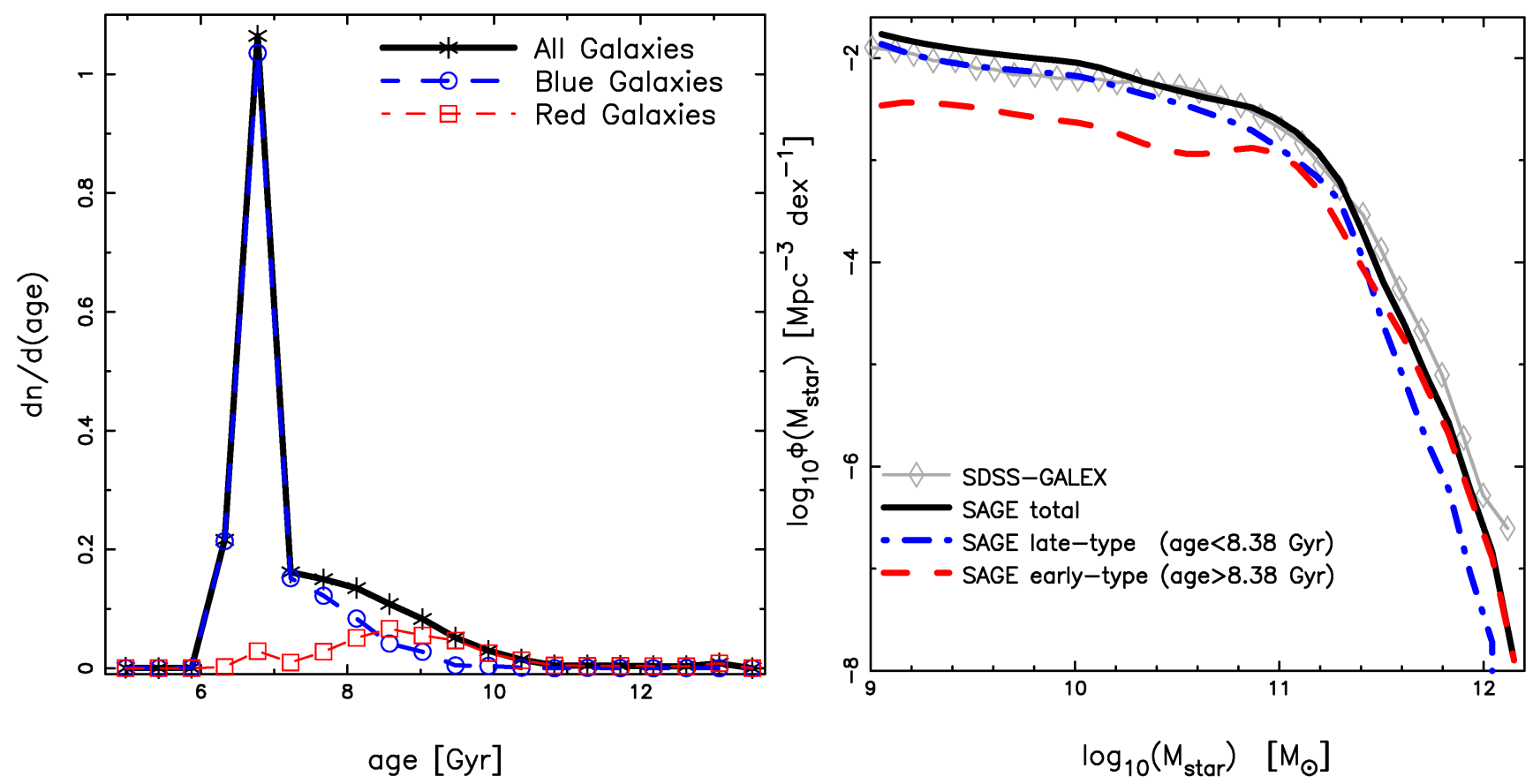

Figure 4. The left panel shows the observed age (mean stellar age in Gyr) distribution of the total (crosses, thick solid line) blue (open circles, thick dashed line) and red (open squares, thin dashed line) sample of galaxies in ALFALFA. The intersection of the age distribution of blue and red galaxies at $t_{\text {age }}=8.38 \mathrm{Gyr}$ is used to classify the blue and red populations as late-type and early-type galaxies respectively. The right panel shows the SMF. The open-diamonds with thin solid line represents observational estimates of the SMF from SDSS-GALEX (Moustakas et al. 2013). The thick solid line is from the SAGE catalog (Knebe et al. 2018) which is based on the MDPL2 simulation (Klypin et al. 2016). The dot-dashed (dashed) line is the SMF for late-type (early-type) galaxies defined as $t_{\text {age }}<8.38\left(t_{\text {age }}>8.38\right)$ Gyr in the context of HI-selected galaxies (see left panel).

ated with the central halo (unless we have mergers of nearly equal masses), due to which the HMF is close to the HMF for centrals. The HMF for satellites contributes little to the HMF.

A similar result is seen observationally for the SMF. The SMF of the centrals dominates the total SMF, whereas satellites contribute little to the total SMF (Drory, et al. 2009). In our definition field halos are centrals without satellites. We will assume that every halo (central or satellite) hosts a single galaxy. Similarly HI is associated with a halo via its galaxy. Although it is rare to find considerable amounts of $\mathrm{HI}$ in massive central galaxies of clusters, a significant fraction of $\mathrm{HI}$ is locked up in satellite galaxies as seen in observations (Lah, et al. 2009). The results of Guo et al. (2020) corroborate this observation. As seen in figure 3 of Guo et al. (2020) the total HI content in satellites can be at least $60 \%$ in a group of mass $M_{\mathrm{h}} \sim 13$. However groups and clusters are less abundant as compared to lower mass objects. We therefore expect (as in the case of the galaxy SMF) that satellites contribute little to the HIMF. This expectation is consistent with semi-analytical models of HI (Kim, et al. 2017).

We use a very large publicly available dark matter simulation - MultiDark Planck 2 (MDPL2) (Klypin et al. 2016). MDPL2 is run with the publicly available code GADGET-2 (Springel 2005). It evolves the matter density field, sampled by $3840^{3}$ dark matter particles in a comoving box of side $L_{\mathrm{box}}=1 \mathrm{Gpc} / h$, to $z=0$. This corresponds to a dark matter mass resolution $m_{\mathrm{DM}}=$ $1.51 \times 10^{9} M_{\odot} / h$. The force resolution is $\epsilon=5 \mathrm{kpc} / h$.
The MDPL2 was run with a flat $\Lambda$ CDM cosmology with $\left(\Omega_{\Lambda}, \Omega_{m}, h, \sigma_{8}, n_{s}\right)=(0.693,0.307,0.678,0.823,0.96)$ consistent with the Planck15 results (Planck Collaboration, Ade et al. 2016). The data products include halo (central + satellite) catalogs and merger trees which were obtained with the help of the ROCKSTAR (Behroozi, Wechsler, \& Wu 2013) and CONSISTENT TREES (Behroozi et al. 2013) codes. The MultiDark-Galaxies (Knebe et al. 2018) is a catalog of galaxies built on the data products of MDPL2 with the semi-analytical model - Semi-Analytic Galaxy Evolution (SAGE). The SAGE model is tuned to reproduce the SMF in the local Universe. Galaxy colors and $r$-band luminosities are not well reproduced in SAGE. SAGE also provides the mean age of the stellar population of the galaxy, $t_{\text {age }}$ which we use as a proxy for color. The stellar mass and age of the galaxy, $M_{\text {star }}$ and $t_{\text {age }}$, are available for our HI sample from kcorrect and SDSS (Conroy, Gunn, \& White 2009; Ahn et al. 2014) respectively, so that we can choose a galaxy population based on $t_{\text {age }}$.

We use the MDPL2 halo catalog to obtain the HMF. This is shown as the thick solid line in the right panel of figure 3 . The downward arrow at $M_{\mathrm{h}} \sim 11$ is the mass resolution of the MDPL2 catalog. We have extrapolated the power-law to obtain the HMF at lower masses. We will next define the HI-selected halo mass function, $\phi^{\mathrm{HI}}\left(M_{\mathrm{h}}\right)$, which reproduces the observations of Guo et al. (2020) (open circles in the left panel of figure 3) by defining it with the 

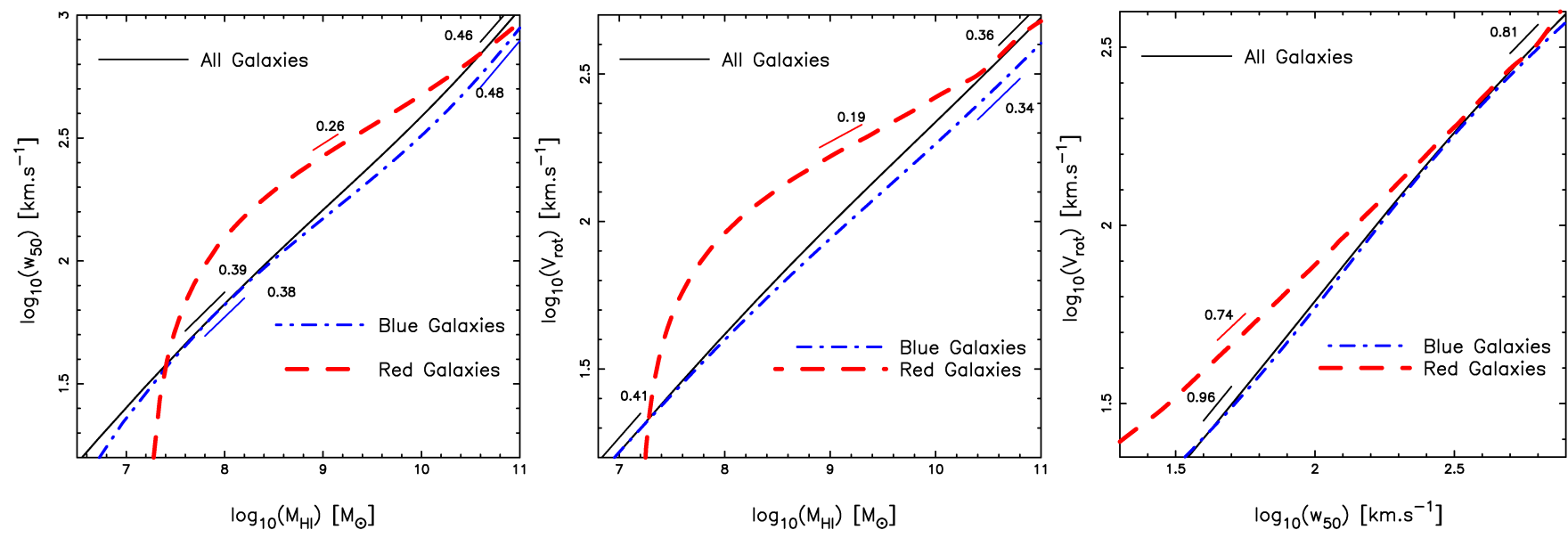

Figure 5. The figure shows the scaling relations by abundance matching the three HI distributions, namely the HIMF, HIWF and HIVF amongst themselves. The left, middle and right panels show the scaling relation $M_{\mathrm{HI}}-w_{50}, M_{\mathrm{HI}}-V_{\text {rot }}^{\mathrm{HI}}$ and $w_{50}-V_{\text {rot }}^{\mathrm{HI}}$ respectively for the total (solid), red(dashed) and blue (dot-dashed) samples.

parametric form:

$$
\phi^{\mathrm{HI}}\left(M_{\mathrm{h}}\right)=\phi\left(M_{\mathrm{h}}\right) \times \frac{f}{1+\left(\frac{M_{\mathrm{h}}}{M_{\mathrm{h}}^{*}}\right)^{\gamma}}
$$

where $\phi\left(M_{\mathrm{h}}\right)$ is the halo mass function. $f$ is an overall fraction that reduces the abundances $\phi^{\mathrm{HI}}\left(M_{\mathrm{h}}\right)$ with respect to $\phi\left(M_{\mathrm{h}}\right)$ and the abundance is further reduced by $\left(\frac{M_{\mathrm{h}}}{M_{\mathrm{h}}^{*}}\right)^{\gamma}$ for $M_{\mathrm{h}} \gtrsim M_{\mathrm{h}}^{*}$. This is a three-parameter functional form that is justified by the various HI observations described earlier. However we point out that it is by no means unique. One can further suppress the HI mass at lower halo masses (Bagla, Khandai, \& Datta 2010; Padmanabhan, Refregier, \& Amara 2017) since these low mass halos would host negligible amounts of HI due to the ionizing background. However the suppression is expected to happen at circular velocities smaller than $30 \mathrm{~km} / \mathrm{s}$, which correspond to the smallest HI detections in ALFALFA $\left(M_{\mathrm{HI}} \sim 7.0-7.5\right)$ and is naturally taken care of by the ALFALFA selection function.

We finally need to fix $\phi^{\mathrm{HI}}\left(M_{\mathrm{h}}\right)$ (eq. 9) described by the three parameters $\left\{f, M_{\mathrm{h}}^{*}, \gamma\right\}$ so as to match the mean observed scaling relation $\left\langle M_{\mathrm{HI}}\right\rangle-M_{\mathrm{h}}$ for centrals of Guo et al. (2020). As discussed earlier we will not distinguish between centrals and satellites while abundance matching. HI is assigned based on halo mass and does not depend whether the halo is a central or satellite. Guo et al. (2020) do not have a corresponding estimate of $\left\langle M_{\mathrm{HI}}^{\mathrm{sat}}\right\rangle-M_{\mathrm{h}}$, but rather have $\left\langle M_{\mathrm{HI}}^{\mathrm{sat}}{ }^{\text {tot }}\right\rangle-M_{\mathrm{h}}$, where $\left\langle M_{\mathrm{HI}}^{\mathrm{sat}, \text { tot }}\right\rangle$ is the mean total HI mass in satellites hosted in centrals of mass $M_{\mathrm{h}}$. The parameters $\left\{f, M_{\mathrm{h}}^{*}, \gamma\right\}$ are fixed by $\chi^{2}$ minimization, so that the scaling relation between $M_{\mathrm{HI}}$ and $M_{\mathrm{h}}$ at fixed halo mass (obtained by abundance matching $\phi\left(M_{\mathrm{HI}}\right)$ to $\left.\phi^{\mathrm{HI}}\left(M_{\mathrm{h}}\right)\right)$, when averaged over all halos in the mass range $\left[M_{\mathrm{h}}, M_{\mathrm{h}}+d M_{\mathrm{h}}\right]$ (given by the total HMF, $\phi\left(M_{\mathrm{h}}\right)$ ) reproduces the observed points of Guo et al. (2020) (open circles in the left panel of figure 3). The choice of $\left\{f=0.320, M_{\mathrm{h}}^{*}=13.661, \gamma=0.996\right\}$, obtained by this minimization procedure, results in the solid line in the left panel of figure 3. This constrains the HIselected HMF, $\phi^{\mathrm{HI}}\left(M_{\mathrm{h}}\right)$, and is shown as the thin solid line in the right panel of figure 3.

If we abundance match the HIMF to the HI-selected
HMF we obtain a scaling relation (dotted line) in the left panel of figure 3 . The dashed line is obtained by abundance matching the HIMF to the HMF. Since $\phi^{\mathrm{HI}}\left(M_{\mathrm{h}}\right)$ represents a subsample of all halos (described by $\phi\left(M_{\mathrm{h}}\right)$ ), HI is now distributed in a smaller number of halos, thereby increasing the HI mass at fixed halo mass. This results in a HI-selected scaling relation (dotted line) above the halo mass selected scaling relation (dashed line). The HI selected scaling relation (dotted line) is well described by a double power law

$$
M_{\mathrm{HI}}\left(M_{\mathrm{h}}\right)=M_{\mathrm{HI}}^{\mathrm{A}} \frac{\left(\frac{M_{\mathrm{h}}}{M_{\mathrm{ht}}}\right)^{\alpha}}{\left[1+\left(\frac{M_{\mathrm{h}}}{M_{\mathrm{ht}}}\right)^{\beta}\right]}
$$

Here $M_{\mathrm{HI}}^{\mathrm{A}}$ is the amplitude, $\alpha$ is the slope of the scaling relation at lower masses which gets suppressed to a slope of $\alpha-\beta$ at masses greater than a transition halo mass, $M_{\mathrm{ht}} \ll M_{\mathrm{h}}$. We find $\left(M_{\mathrm{HI}}^{\mathrm{A}}, \alpha, \beta, M_{\mathrm{ht}}\right)=(9.59,2.10,1.76,10.62)$ describes well the HI selected $M_{\mathrm{HI}}-M_{\mathrm{h}}$ scaling relation in figure 3 .

Since we are working with the red and blue populations amongst the HI-selected galaxies we would also like to obtain corresponding scaling relations for these populations as well. This is where the SAGE catalog becomes useful. However, the SAGE catalog does not have accurate estimates of colors and magnitudes that are needed to determine the red and blue populations. We therefore need to come up with an approximate proxy for the red and blue populations. Apart from rest-frame magnitudes, kcorrect also provides estimates of the stellar mass and the mean age of the stellar population, $t_{\text {age }}$, of the galaxy is obtained from the Granada FSPS models (Conroy, Gunn, \& White 2009; Ahn et al. 2014) from SDSS. In the left panel of figure 4 we show the age distribution of the red and blue samples in ALFALFA. The age distribution of the blue population has a pronounced peak at $t_{\text {age }} \sim 6.8 \mathrm{Gyr}$ and drops rapidly beyond $t_{\text {age }}>7.2 \mathrm{Gyr}$. The age distribution of the red population has a peak at $t_{\text {age }} \sim 8.5 \mathrm{Gyr}$ and the distribution is broad. Although, bimodal, the distribution is suppressed for red galaxies since ALFALFA primarily samples the blue cloud. In spite of this we can see that the red population in ALFALFA is an older, early-type population compared to the blue population. The 

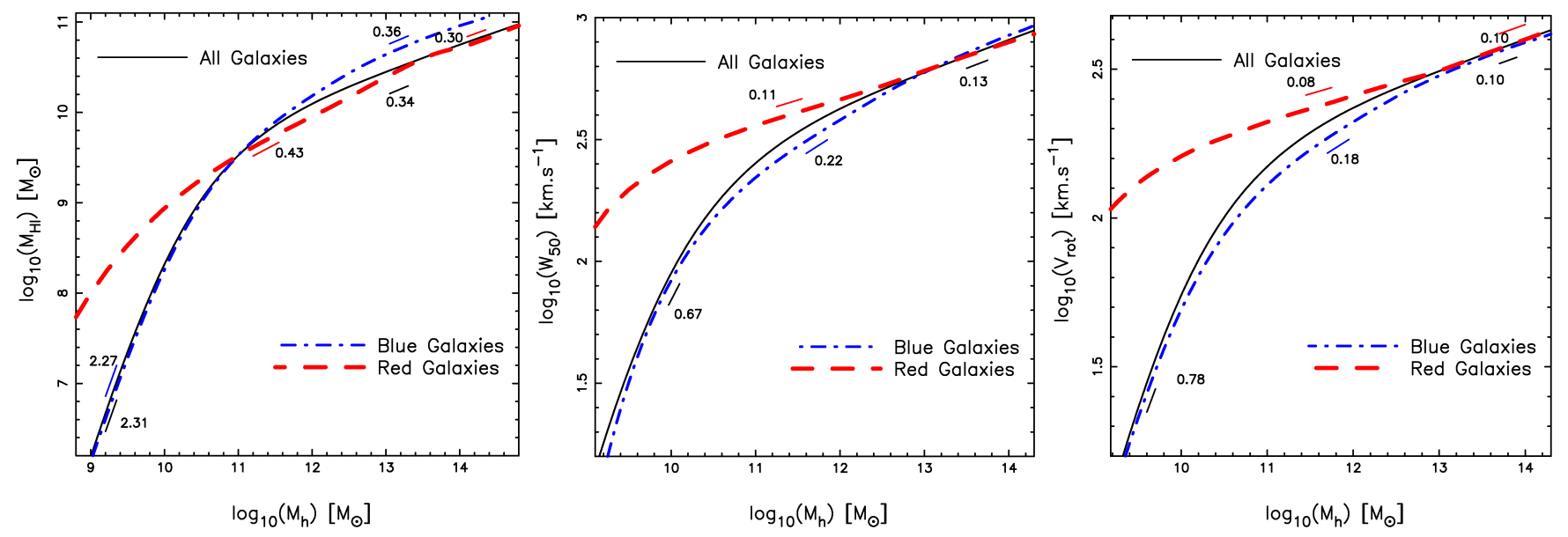

Figure 6. The figure shows the scaling relations by abundance matching the three HI distributions, namely the HIMF, HIWF and HIVF with the HI-selected HMF (equation 9). The left, middle and right panels show the scaling relation $M_{\mathrm{h}}-M_{\mathrm{HI}}, M_{\mathrm{h}}-w_{50}$ and $M_{\mathrm{h}}-V_{\text {rot }}^{\mathrm{HI}}$ respectively for the total (solid), red(dashed) and blue (dot-dashed) samples.

distributions intersect at $t_{\text {age }}=8.38 \mathrm{Gyr}$. We can therefore use $t_{\text {age }}$, as a rough proxy for colors, with $t_{\text {age }}>8.38 \mathrm{Gyr}$ $\left(t_{\text {age }}<8.38 \mathrm{Gyr}\right)$ representing the red (blue) populations. This definition has been made on the basis of the ALFALFA (HI-selected) sample. A similar definition could be made on the basis of a stellar mass selected sample, from SDSS. However we wish to use the HI-selected HMF to abundance match to the HI distributions therefore we will stick with this definition.

We use this criterion to identify galaxies in SAGE as early (red) or late (blue) type galaxies. The SAGE SMF (solid line) is plotted in the right panel of figure 4. It compares well with the observed SMF (open diamonds, thin line) from SDSS-GALEX (Moustakas et al. 2013). The dashed (dot-dashed) line is the contribution from red, early-type (blue, late-type) galaxies to the SMF from SAGE. The red population dominates the high mass end of the SMF whereas the blue population dominates the SMF at lower masses. The bimodality is however not as distinct since the classification was done based on an HI-selected ALFALFA sample. If it were done on a stellar mass selected sample a clear bimodality is seen (Baldry, et al. 2012)

In the right panel of figure 3 we plot the HMF corresponding to red (early-type) and blue (late-type) galaxies as thick dashed and thick dot-dashed lines respectively. The corresponding HI-selected HMF are plotted with thin lines. One can see that the early-type galaxies dominate the HMF, and the HI-selected HMF, at $M_{\mathrm{h}} \gtrsim 12.2$ and the late-type galaxies dominate below this mass.

We are now in a position to obtain scaling relations between various $\mathrm{HI}$ properties by abundance matching the HIMF, HIWF and the HIVF to each other. Having defined and constrained the HI-selected HMF, $\phi^{\mathrm{HI}}\left(M_{\mathrm{h}}\right)$, we can also abundance match the HIMF, HIWF and HIVF to the HIselected HMF.

In figure 5 we show the scaling relations $M_{\mathrm{HI}}-w_{50}$, $M_{\mathrm{HI}}-V_{\mathrm{rot}}^{\mathrm{HI}}$ and $w_{50}-V_{\mathrm{rot}}^{\mathrm{HI}}$ in the left, middle and right panels respectively. These relations were obtained by abundance matching the HIMF-HIWF, HIMF-HIVF and HIWF-HIVF. This is done for the total (solid line), red (dashed line) and blue (dot-dashed line) samples. The scaling relation for the total sample can be thought as a galaxy-count weighted sum of the scaling relations of the red and blue samples. Since HI is primarily sampled by the blue cloud, the scaling relation for the full sample is closer to the scaling relation of blue galaxies. The scaling relations $M_{\mathrm{HI}}-w_{50}$ and $M_{\mathrm{HI}}-V_{\text {rot }}^{\mathrm{HI}}$ are different for the red and blue samples. At lower masses the HI detections are primarily in the blue cloud, we therefore see a rapid drop in the scaling relations below $M_{\mathrm{HI}}=7.6$ for the red sample. For $M_{\mathrm{HI}} \in[8.5,10]$ we find that at fixed HI mass the red sample has larger velocity profile widths. This suggests that in this range the red sample is on average hosted in larger halos because the profile width (or rotational velocity ) is a good proxy for the halo mass. Although we have not invoked the HI-selected HMF at this stage, we see that this explanation is consistent with figure 3 and figure 6 . In the right panel of figure 5 we see the $w_{50}-V_{\text {rot }}^{\mathrm{HI}}$ scaling relation for the red sample is above that of the blue sample at lower velocities, but they asymptote to each other at larger velocities.

In figure 6 we show the scaling relations $M_{\mathrm{h}}-M_{\mathrm{HI}}$ (left panel), $M_{\mathrm{h}}-w_{50}$ (middle panel) and $M_{\mathrm{h}}-V_{\text {rot }}^{\mathrm{HI}}$ (right panel) which were obtained by abundance matching the HI-selected HMF-HIMF, HI-selected HMF-HIWF and HIselected HMF-HIVF respectively. This was done separately for the total (solid line), red (dashed line) and blue (dotdashed line) samples. The $M_{\mathrm{h}}-M_{\mathrm{HI}}$ scaling relation is qualitatively similar in shape to the $M_{\mathrm{h}}-M_{\text {star }}$ scaling relation (Behroozi, Conroy, \& Wechsler 2010; Behroozi et al. 2019) and is described by a double power-law: a steep power law with slope $\sim 2.1$ at lower masses transitioning to a shallower power law with slope $\sim 0.34$ above masses $M_{\mathrm{h}} \gtrsim 10.6$ (see equation 10). The transition mass $M_{\mathrm{ht}}=10.62$ for $M_{\mathrm{h}}-M_{\mathrm{HI}}$, is more than an order of magnitude smaller than the transition mass of $M_{\mathrm{h}} \simeq 12$ (Behroozi et al. 2019) for the $M_{\mathrm{h}}-M_{\text {star }}$ relation. This suggests that baryonic processes like heating and feedback in larger mass halos suppress HI gas on a shorter time scale compared to star-formation. A double power-law is also seen in the scaling relations for $M_{\mathrm{h}}-w_{50}$ and $M_{\mathrm{h}}-V_{\mathrm{rot}}^{\mathrm{HI}}$ with the transition in slopes occurring at $M_{\mathrm{h}} \sim 11$. As in figure 5 the scaling relation of the total sample is close to that of the blue sample. 


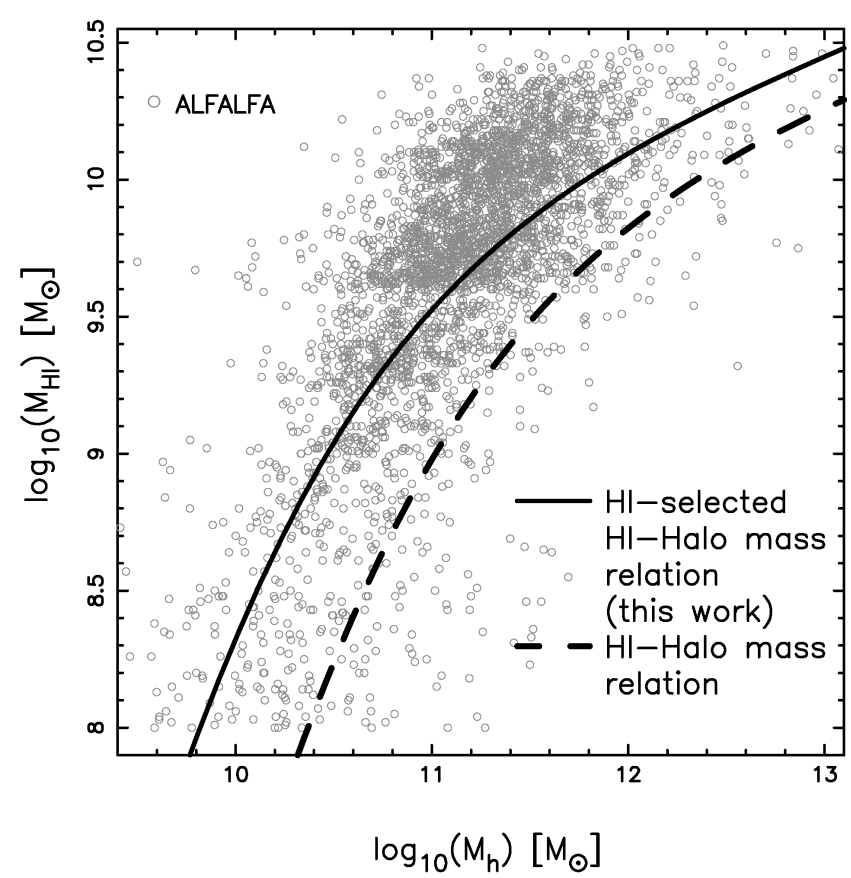

Figure 7. The $M_{\mathrm{HI}}-M_{\mathrm{h}}$ relation (open circles) for a volumelimited subsample generated from the ALFALFA survey. The $M_{\mathrm{h}}$ masses were estimated using the $M_{\mathrm{star}}-M_{\mathrm{h}}$ relation of Moster, Naab, \& White (2013). The solid (dashed) line is the $M_{\mathrm{HI}}-M_{\mathrm{h}}$ relation obtained by abundance matching the HIMF with the HIselected HMF (HMF).

At the low mass end $\left(M_{\mathrm{h}} \leq 11\right)$ of the $M_{\mathrm{h}}-M_{\mathrm{HI}}$ scaling relation we find that at fixed halo the red sample is richer in HI compared to their blue counterparts. The situation is reversed at the high mass end. At the high mass end, we can turn this around. We find at fixed $M_{\mathrm{HI}}$ the halo mass is larger for the red sample compared to the blue sample. In the middle and right panels we see a similar trend for $M_{\mathrm{h}}-w_{50}$ and $M_{\mathrm{h}}-V_{\text {rot }}^{\mathrm{HI}}$ at lower masses. However at larger masses the relations of the blue and red samples asymptote to each other, suggesting that the velocity profile is a good descriptor of the halo mass irrespective of galaxy type.

We end this section by comparing the $M_{\mathrm{HI}}-M_{\mathrm{h}}$ relation obtained with ALFALFA data. This is a useful consistency check of our results with data. We create five volume limited samples in equal bins of mass from ALFALFA, in the mass range $M_{\mathrm{HI}} \in[8.0,10.5[$. The five volume limited samples are disjoint sets. We combine them to create a final volume limited sample. We use the stellar masses of these galaxies and convert them into halo masses using the tight $M_{\mathrm{star}}-M_{\mathrm{h}}$ scaling relation of Moster, Naab, \& White (2013). Using other relations, e.g. Behroozi, Conroy, \& Wechsler (2010); Behroozi et al. (2019) does not result in significant changes to our result in figure 7 . The solid line is the scaling relation that we obtain by abundance matching the HIMF to the HI-selected HMF (equation 9) and the dashed line is the scaling relation obtained by abundance matching the HIMF to the HMF. Clearly the scaling that we obtain (solid line) is in better agreement with the data as compared to the scaling relation obtained by abundance matching the HIMF and HMF (dashed line).

\section{DISCUSSION AND SUMMARY}

In this work we have used data from the ALFALFA survey to obtain the HIMF, HIWF and HIVF for HI-selected galaxies. The survey volume that we have considered overlaps with SDSS and allows us to also look at these abundances for the red and blue population of galaxies. We then use recent observations from ALFALFA which estimate $\left\langle M_{\mathrm{HI}}\right\rangle$ - $M_{\mathrm{h}}$ relation in massive centrals (Guo et al. 2020) to finally estimate an HI-selected $\mathrm{HMF}, \phi^{\mathrm{HI}}\left(M_{\mathrm{h}}\right)$ (equation 9). $\phi^{\mathrm{HI}}\left(M_{\mathrm{h}}\right)$ is parameterized by three parameters which are fixed to match the observed $\left\langle M_{\mathrm{HI}}\right\rangle-M_{\mathrm{h}}$ relation. Although an upper bound, this relation explains the $M_{\mathrm{HI}}-M_{\mathrm{h}}$ relation for an HI-selected sample (figure 7). We then use a semianalytic galaxy catalog, SAGE, which was generated from a large simulation, MDPL2, to further obtain the HI-selected HMF for red (early-type) and blue(late-type) galaxies.

There are a number of assumptions while obtaining the HI-selected HMF for red and blue galaxies. We have assumed that age is a proxy for color, justified observationally (figure 4). Although it gives a qualitatively similar bimodal behavior to that seen in the observed (Drory, et al. 2009; Baldry, et al. 2012) SMF (figure 4), it is by no way an exact proxy. The second assumption is that the stellar ages from SAGE are accurate. The stellar ages from SAGE are based on the halo merger trees (or growth histories) and the various assumptions of their model. In spite of this it gives a qualitatively and physically reasonable bimodal distribution for age. Finally we have used the same relation (equation 9) to obtain an HI selected halo mass function for both the red and blue galaxies. This may not be true. In order to distinguish between them we would need the stacking results of Guo et al. (2020) to be made for red and blue galaxies separately which is not available. With these assumptions in mind we stress that the HI-halo scaling relations for the red and blue sample may not be completely accurate, but should be thought of as a result which should be revisited once more data (both from observations and simulations) becomes available in the future. However the various HI scaling relations (figure 5) are robust for the total, red and blue samples since there are no model assumptions. Similarly the HI-halo scaling relations for the total sample are also robust.

Recently Li et al. (2019a) presented the HI-selected HMF for late-type galaxies. They used a scaling relation $w_{50}-M_{\mathrm{h}}$ from 175 late-type galaxies in the Spitzer Photometry and Accurate Rotation Curves (SPARC, Lelli, McGaugh, \& Schombert 2016) catalog to determine the halo mass function of early-type HI-selected galaxies in HIPASS. The 2DSWML method was used to obtain the HI-selected HMF for early type galaxies by binning in $M_{\mathrm{h}}$ instead of $w_{50}$ or $V_{\text {rot }}$. The SPARC catalog uses near-infrared (NIR) Spitzer photometry $(3.6 \mu \mathrm{m})$ to trace the stellar mass distribution in galaxies. This is important to break the star-halo degeneracy (Lelli, McGaugh, \& Schombert 2016) when mass modeling galaxies. Additionally it relies on $\mathrm{HI} / \mathrm{H} \alpha$ rotation curve measurements over the past 3 decades. The rotation curves are finally fit assuming a halo profile, which results in a halo mass $M_{200} \equiv M_{\mathrm{h}}$ estimate of the galaxy (Li et al. 2019b, 2020). The halo mass estimates of $\mathrm{Li}$ et al. (2019b) were made after imposing the halo mass - concentration relation (Dutton \& Macciò 2014) and the stellar mass - halo mass relation (Moster, Naab, \& White 2013) as priors. Imposing 

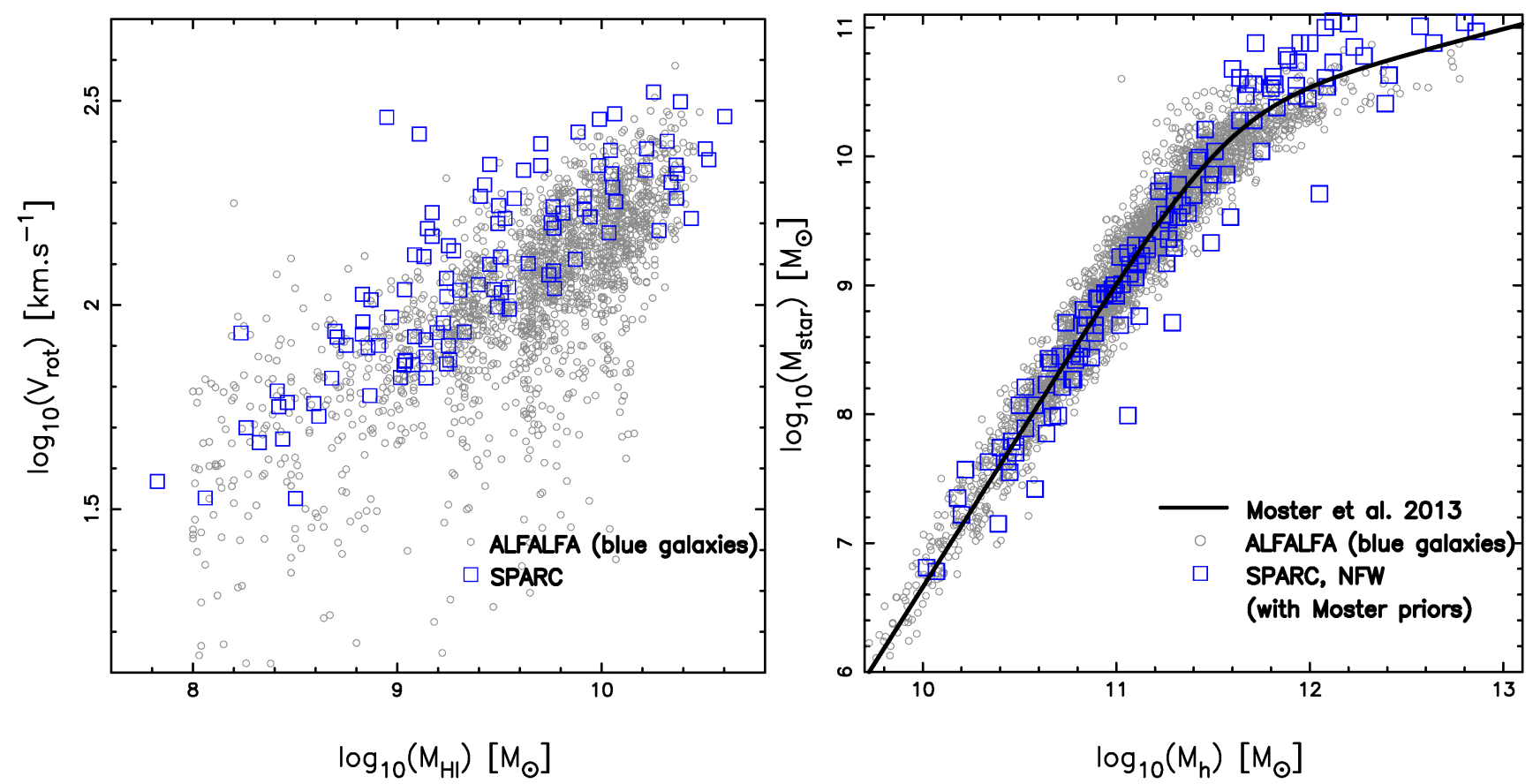

Figure 8. Left Panel: A comparison of HI properties, $M_{\mathrm{HI}}-V_{\mathrm{rot}}$, for a volume limited sample of blue galaxies in ALFALFA (open circle) and SPARC (open square). Right panel: Same as left panel but for $M_{\mathrm{h}}-M_{\mathrm{star}}$. The solid line is the $M_{\mathrm{star}}-M_{\mathrm{h}}$ relation of Moster, Naab, \& White (2013).
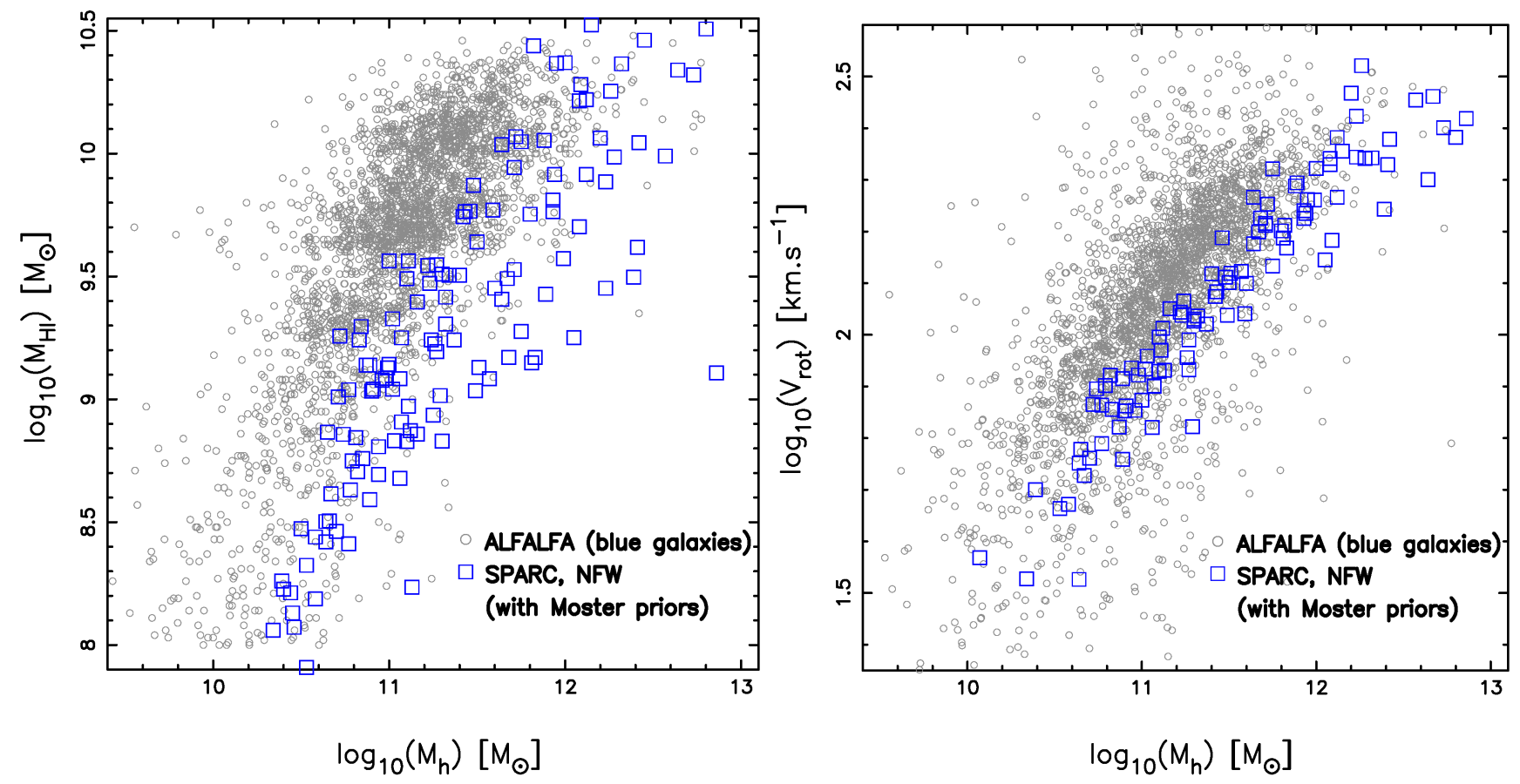

Figure 9. Left Panel: A comparison of $M_{\mathrm{HI}}-M_{\mathrm{h}}$, for a volume limited sample of blue galaxies in ALFALFA (open circle) and SPARC (open square). Right panel: Same as left panel but for $V_{\mathrm{rot}}-M_{\mathrm{h}}$.

these priors reduces the scatter between the halo mass and other properties of the galaxy like $M_{\mathrm{star}}, M_{\mathrm{HI}}, V_{\mathrm{rot}}, w_{50}$. We will consider halo mass estimates with these priors imposed in order to be consistent with $\mathrm{Li}$ et al. (2019a). We however point out that $\mathrm{Li}$ et al. (2020) have also released halo mass estimates which relax these assumptions. The system- atic differences between SPARC and ALFALFA (figure 9) which we discuss next, persist nevertheless, irrespective of halo mass estimates. Although the halo mass estimates exist for many profiles, we will only consider the estimate based on the NFW (Navarro, Frenk, \& White 1996) profile. Finally SPARC extracts profile widths, $w_{50}$ of these 
galaxies from the Extragalactic Distance Database (Tully et al. 2009; Courtois et al. 2009). The galaxy properties e.g. $M_{\mathrm{star}}, M_{\mathrm{h}}, M_{\mathrm{HI}}, V_{\text {rot }}, w_{50}$ in SPARC forms a near homogeneous data set (Lelli, McGaugh, \& Schombert 2016).

In figure 8 we compare the $M_{\mathrm{HI}}-V_{\text {rot }}$ and the $M_{\mathrm{h}}-M_{\text {star }}$ relation between a volume limited sample of blue galaxies in ALFALFA (open circle) and SPARC (open square). We take the same volume limited sample as shown in figure 7 and choose galaxies with inclinations $i<45^{\circ}$ to reliably obtain $V_{\text {rot }}$ (Zwaan, et al. 2010) from $w_{50}$ after correcting for inclination. The volume limited sample is the same in figure 8 and 9. However in the figures which involve $V_{\text {rot }}$ the sample is smaller since it excludes galaxies with $i>45^{\circ}$. The scaling of HI properties (left panel) between ALFALFA and SPARC agree with each other. The SPARC sample is also homogeneously sampling the range of $M_{\mathrm{HI}}-V_{\text {rot }}$ covered by ALFALFA. In the right panel of figure 8 we compare the $M_{\text {star }}-M_{\mathrm{h}}$ relation between the ALFALFA and SPARC samples. For ALFALFA we do not have an independent measure of $M_{\mathrm{h}}$, we have therefore used the $M_{\mathrm{star}}-M_{\mathrm{h}}$ scaling relation from Moster, Naab, \& White (2013) (solid line) to convert $M_{\text {star }}$ into $M_{\mathrm{h}}$ after accounting for scatter. The SPARC sample agrees with the Moster, Naab, \& White (2013) relation and therefore with the ALFALFA points, confirming again that this relation is tight. Here too we see that SPARC homogeneously samples the $M_{\text {star }}-M_{\mathrm{h}}$ range and the scaling is broadly consistent with ALFALFA.

We now compare the $M_{\mathrm{HI}}-M_{\mathrm{h}}$ and the $V_{\text {rot }}-M_{\mathrm{h}}$ relation between ALFALFA and SPARC in the left and right panels of figure 9 . We see a marked difference between ALFALFA and SPARC in both these figures. We point out that if we replaced $M_{\mathrm{h}}$ with $M_{\text {star }}$ the systematic differences remain. Clearly the joint distribution between an HI property $\left(M_{\mathrm{HI}}\right.$ or $\left.V_{\text {rot }}\right)$ and optical property $\left(M_{\text {star }}\right.$ which is a proxy for $M_{\mathrm{h}}$ ) is different between ALFALFA and SPARC. Although SPARC homogeneously samples individual properties, it is clearly biased compared to ALFALFA. For ALFALFA the selection function is well understood, however SPARC does not have a corresponding selection function since it relies on individual objects. At fixed, $M_{\mathrm{HI}}$ or $V_{\text {rot }}$ SPARC predicts a larger halo mass. We would therefore expect the HI-selected HMF for late-type galaxies using the SPARC scaling $\left(M_{\mathrm{h}}-V_{\text {rot }}\right.$ or $\left.M_{\mathrm{h}}-M_{\mathrm{HI}}\right)$, to be offset towards larger halo masses when compared to our result.

This is shown in figure 10 . The estimate by $\mathrm{Li}$ et al. (2019a) is offset by about 0.7 dex towards the right at lower masses and has a sharper exponential drop compared to our results (thin line). The offset is consistent with the offset of 0.77 dex which we estimate from the left panel of figure 9 . However the sharp drop at larger masses cannot be reconciled with our results by simple scaling arguments. We leave the investigation of such issues to future work.

We summarize our results below.

- We have shown that the HIWF and HIVF are described by modified Schechter functions. Both these abundances are well separated for the red and blue populations at larger velocities. The red population dominates the high velocity end and the blue population dominates the velocity function at the knee and lower velocities.

- A qualitatively similar result is seen for the HIMF. However unlike the HIWF and HIVF we find that the differences

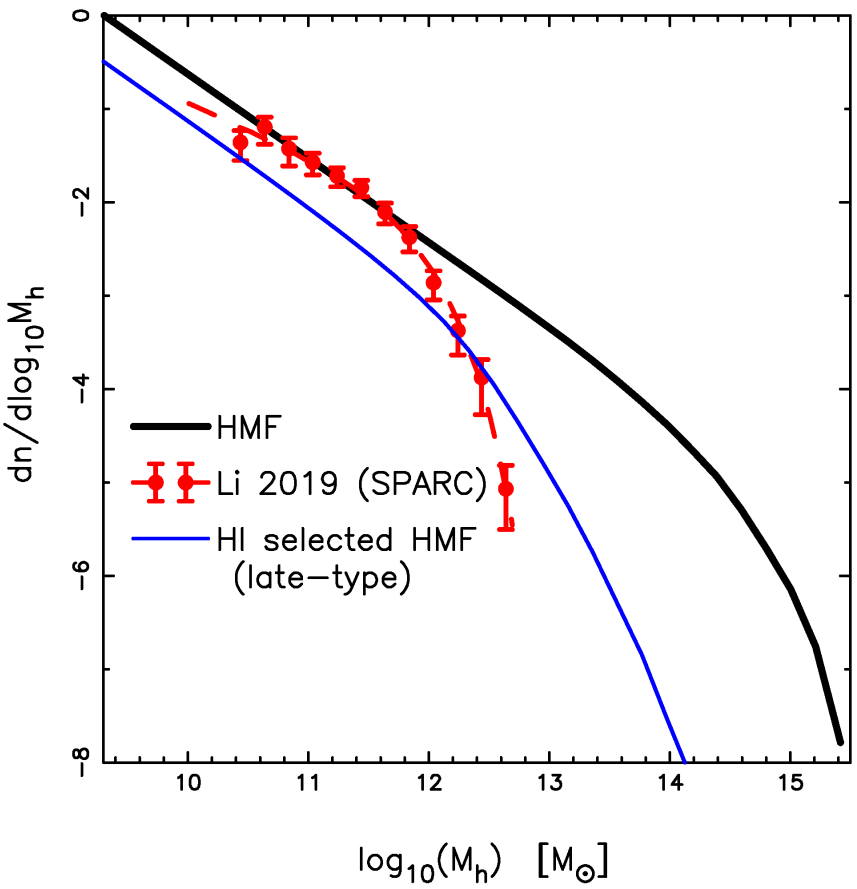

Figure 10. The thick line is the HMF. The thin line is our estimate of the HI-selected HMF for late-type (or blue) galaxies from ALFALFA. The dashed line is the HI-selected HMF for late-type galaxies by $\mathrm{Li}$ et al. (2019a) based on SPARC and HIPASS data. These halo mass estimates are based on the NFW (Navarro, Frenk, \& White 1996) profile, and assume the halo mass-concentration relation (Dutton \& Macciò 2014) and the stellar-halo mass relation (Moster, Naab, \& White 2013) as priors.

in the HIMF for the red and blue populations, at the high mass end, is less pronounced.

- Using the recent observational $\left\langle M_{\mathrm{HI}}\right\rangle-M_{\mathrm{h}}$ relation of Guo et al. (2020) We have estimated the HI-selected HMF (equation 9) which represents the abundances of halos based on HI mass. Using semi-analytic model - SAGE- based galaxy catalog, we have also estimated the HI-selected HMF for the red and blue galaxies.

- Using these six abundances which describe HI rich galaxies we have obtained scaling relations between HI properties $\left(M_{\mathrm{HI}}-w_{50}-V_{\text {rot }}\right)$ and HI-halo properties $M_{\mathrm{HI}}-M_{\mathrm{h}}$, $w_{50}-M_{\mathrm{h}}$ and $V_{\text {rot }}-M_{\mathrm{h}}$ (See figures 5 and 6).

- The $M_{\mathrm{HI}}-M_{\mathrm{h}}$ scaling relation is robust and consistent with a volume limited sample in ALFALFA. It is described by a steep power law slope $\sim 2.10$ at small masses and transitions to a shallower slope $\sim 0.34$ at masses larger than $M_{\mathrm{ht}}=10.62$. It has a shape similar to the $M_{\mathrm{star}}-M_{\mathrm{h}}$ (Behroozi et al. 2019) but the transition halo mass, $M_{\mathrm{ht}}$ scale is smaller by about 1.4 dex compared to that of the $M_{\text {star }}-M_{\mathrm{h}}$ relation. This suggests that baryonic processes like heating and feedback suppress the HI content in large mass halos on a shorter timescale as compared to starformation.

In this work we have obtained scaling relations of $\mathrm{HI}$ selected galaxies. The scaling relations among HI properties, i.e. $M_{\mathrm{HI}}-w_{50}-V_{\mathrm{rot}}^{\mathrm{HI}}$, have been obtained from the same sample with no model assumptions. Although figure 7 shows that our results are consistent with the data (and provides for a consistency check of the approach taken in 
this work) we expect scatter to flatten these relations and the relations between $\mathrm{HI}$ properties and halo mass at the high mass end (Behroozi, Conroy, \& Wechsler 2010; Ren, Trenti, \& Di Matteo 2020). In summary we have effectively constrained a multivariate HI halo model, but unlike traditional approaches in constructing a halo model we have not used HI clustering to constrain it, but have rather constrained the HI - halo mass scaling relations from the results of Guo et al. (2020). In a forthcoming paper we will present our clustering predictions of HI selected galaxies based on the current work.

\section{ACKNOWLEDGMENTS}

We would like to thank the referee for comments and suggestions which have improved the presentation of the paper. We would like to thank Raghunathan Srianand, Aseem Paranjape and Jasjeet Singh Bagla for useful discussions. SD would like to thank Somnath Bharadwaj for useful comments. NK acknowledges the support of the Ramanujan Fellowship ${ }^{1}$ and the IUCAA ${ }^{2}$ associateship programme. All the analyses were done on the XANADU and CHANDrA servers funded by the Ramanujan Fellowship. SD would like to thank Sudhakar Panda for financial support from the J.C. Bose Fellowship ${ }^{1}$.

We thank the entire ALFALFA collaboration in observing, flagging, and extracting the properties of galaxies that this paper makes use of. This work also uses data from SDSS DR7. Funding for the SDSS and SDSS-II has been provided by the Alfred P. Sloan Foundation, the Participating Institutions, the National Science Foundation, the U.S. Department of Energy, the National Aeronautics and Space Administration, the Japanese Monbukagakusho, the Max Planck Society, and the Higher Education Funding Council for England. The SDSS Website is http://www.sdss.org/. The SDSS is managed by the Astrophysical Research Consortium for the Participating Institutions.

The CosmoSim database used in this paper is a service by the Leibniz-Institute for Astrophysics Potsdam (AIP). The MultiDark database was developed in cooperation with the Spanish MultiDark Consolider Project CSD200900064. The authors gratefully acknowledge the Gauss Centre for Supercomputing e.V. (www.gauss-centre.eu) and the Partnership for Advanced Supercomputing in Europe (PRACE, www.prace-ri.eu) for funding the MultiDark simulation project by providing computing time on the GCS Supercomputer SuperMUC at Leibniz Supercomputing Centre (LRZ, www.lrz.de). The Bolshoi simulations have been performed within the Bolshoi project of the University of California High-Performance AstroComputing Center (UCHiPACC) and were run at the NASA Ames Research Center.

1 Awarded by the Department of Science and Technology, Government of India

2 Inter University Centre for Astronomy and Astrophysics, Pune, India

\section{DATA AVAILABILITY}

The data used in this work is publicly available. SDSS DR7 (Abazajian, et al. 2009) data can be accessed from sciserver.org and the $\alpha .40$ (Haynes, et al. 2011) data from ALFALFA can be accessed from egg.astro.cornell.edu. The MultiDark simulations and galaxy catalogs are available in the CosmoSim database at https://www.cosmosim.org.

\section{REFERENCES}

Abazajian, K. N., et al. 2009,ApJS,182, 543

Planck Collaboration, Ade P. A. R., Aghanim N., Arnaud M., Ashdown M., Aumont J., et al., 2016, A\&A, 594, A13.

Ahn C. P., Alexandroff R., Allende Prieto C., Anders F., Anderson S. F., et al., 2014, ApJS, 211, 17.

Bagla J. S., Khandai N., Datta K. K., 2010, MNRAS, 407, 567.

Baldry, I. K., et al. 2004, ApJ,600,681

Baldry et al., 2012, MNRAS, 421, 621

Behroozi P. S., Conroy C., Wechsler R. H., 2010, ApJ, 717, 379 .

Behroozi P. S., Wechsler R. H., Conroy C., 2013, ApJ, 770, 57.

Behroozi P. S., Wechsler R. H., Wu H.-Y., 2013, ApJ, 762, 109.

Behroozi P. S., Wechsler R. H., Wu H.-Y., Busha M. T., Klypin A. A., Primack J. R., 2013, ApJ, 763, 18.

Behroozi P., Wechsler R. H., Hearin A. P., Conroy C., 2019, MNRAS, 488, 3143.

Blanton M. R., Roweis S., 2007, AJ, 133, 734

Casey C. M., Narayanan D., Cooray A., 2014, PhR, 541, 45

Carilli C. L., Walter F., 2013, ARA\&A, 51, 105.

Catinella B., et al., 2013, MNRAS, 436, 34

Chae K.-H., 2010, MNRAS, 402, 2031.

Chaves-Montero J., Angulo R. E., Schaye J., Schaller M., Crain R. A., Furlong M., Theuns T., 2016, MNRAS, 460, 3100 .

Conroy C., Wechsler R. H., 2009, ApJ, 696, 620.

Conroy C., Gunn J. E., White M., 2009, ApJ, 699, 486.

Courtois H. M., Tully R. B., Fisher J. R., Bonhomme N., Zavodny M., Barnes A., 2009, AJ, 138, 1938.

Crain R. A., Schaye J., Bower R. G., Furlong M., Schaller M., et al., 2015, MNRAS, 450, 1937.

Crain R. A., Bahé Y. M., Lagos C. del P., Rahmati A., Schaye J., et al., 2017, MNRAS, 464, 4204.

Davé R., Anglés-Alcázar D., Narayanan D., Li Q., Rafieferantsoa M. H., Appleby S., 2019, MNRAS, 486, 2827.

Davé R., Crain R. A., Stevens A. R. H., Narayanan D., Saintonge A., et al., 2020, MNRAS, 497, 146.

Davis M., Huchra J., 1982, ApJ, 254, 437

Diemer B., Stevens A. R. H., Lagos C. del P., Calette A. R., Tacchella S., et al., 2019, MNRAS, 487, 1529.

Di Matteo T., Khandai N., DeGraf C., Feng Y., Croft R. A. C., et al., 2012, ApJL, 745, L29.

Drory N., et al., 2009, ApJ, 707, 1595

Dutta S., Khandai N., Dey B., (D20) 2020, MNRAS, 494, 2664

Dutta S., Khandai N., (D21) 2021, MNRAS, 500, L37

Durbala A., Finn R. A., Crone Odekon M., Haynes M. P., Koopmann R. A., O’Donoghue A. A., 2020, AJ, 160, 271. 
Efstathiou G., Ellis R. S., Peterson B. A., 1988, MNRAS, 232, 431

Feng Y., Di-Matteo T., Croft R. A., Bird S., Battaglia N., et al., 2016, MNRAS, 455, 2778.

Gordon K. J., 1971, ApJ, 169, 235.

Guo H., Li C., Zheng Z., Mo H. J., Jing Y. P., et al., 2017, ApJ, 846, 61.

Guo H., Jones M.G., Haynes M.P., Fu J., 2020, ApJ, 894, 92

Haynes M. P., et al., 2011, AJ, 142, 170

Haynes M. P., et al., 2018 ApJ 861, 49

Huang S., Haynes M. P., Giovanelli R., Brinchmann J., 2012, ApJ, 756, 113

Kennicutt R. C., 1989, ApJ, 344, 685

Kennicutt R. C., 1998, ApJ, 498, 541

Kim H.-S. et al., 2017, MNRAS, 465, 111

Khandai N., et al., 2011, MNRAS, 415, 2580

Khandai N., Di Matteo T., Croft R., Wilkins S., Feng Y., et al., 2015, MNRAS, 450, 1349.

Klypin A., Yepes G., Gottlöber S., Prada F., Heß S., 2016, MNRAS, 457, 4340.

Knebe A., Stoppacher D., Prada F., Behrens C., Benson A., Cora S. A., Croton D. J., et al., 2018, MNRAS, 474, 5206.

Lah P., et al., 2009, MNRAS, 399, 1447

Lelli F., McGaugh S. S., Schombert J. M., 2016, AJ, 152, 157.

Lelli F., McGaugh S. S., Schombert J. M., Pawlowski M. S., 2017, ApJ, 836, 152.

Lemonias J. J., Schiminovich D., Catinella B., Heckman T. M., Moran S. M., 2013, ApJ, 776, 74

Li P., Lelli F., McGaugh S., Pawlowski M. S., Zwaan M. A., Schombert J., 2019, ApJL, 886, L11.

Li P., Lelli F., McGaugh S. S., Starkman N., Schombert J. M., 2019, MNRAS, 482, 5106.

Li P., Lelli F., McGaugh S., Schombert J., 2020, ApJS, 247, 31.

Loveday, J., 2000, MNRAS,312,557

Dutton A. A., Macciò A. V., 2014, MNRAS, 441, 3359.

Moster B. P., Naab T., White S. D. M., 2013, MNRAS, 428,3121 .

Navarro J. F., Frenk C. S., White S. D. M., 1996, ApJ, 462, 563.

Madau P., Dickinson M., 2014, ARA\&A, 52, 415.

Maddox N., Hess K. M., Obreschkow D., Jarvis M. J., Blyth

S.-L., 2015, MNRAS, 447, 1610

Martin, A., et al., 2010, ApJ, 723,1359

Meyer M. J., Zwaan M. A., Webster R. L., Staveley-Smith L., Ryan-Weber E., et al., 2004, MNRAS, 350, 1195.

Moorman C. M., et al., 2014, MNRAS, 444, 3559

Moustakas J., Coil A. L., Aird J., Blanton M. R., Cool R. J., Eisenstein D. J., Mendez A. J., et al., 2013, ApJ, 767,50 .

Naab T., Ostriker J. P., 2017, ARA\&A, 55, 59.

Obuljen A., Alonso D., Villaescusa-Navarro F., Yoon I., Jones M., 2019, MNRAS, 486, 5124.

Padmanabhan H., Kulkarni G., 2017, MNRAS, 470, 340

Padmanabhan H., Refregier A., Amara A., 2017, MNRAS, 469, 2323.

Papastergis, E., Martin, A.M., Giovanelli, R. \& Haynes, M.P. 2010, ApJ 739, 38

Paranjape A., Choudhury T. R., Sheth R. K., 2021, MN-
RAS, 503, 4147.

Paranjape A., Srianand R., Choudhury T. R., Sheth R. K., 2021, arXiv, arXiv:2105.04570

Paul N., Choudhury T. R., Paranjape A., 2018, MNRAS, 479, 1627.

Pillepich A., Nelson D., Hernquist L., Springel V., Pakmor R., et al., 2018, MNRAS, 475, 648.

Rahmati A., Schaye J., Pawlik A. H., Raičević M., 2013, MNRAS, 431, 2261.

Ren K., Trenti M., Di Matteo T., 2020, ApJ, 894, 124.

Rhee J., et al., 2018, MNRAS, 473, 1879

Romeo A. B., 2020, MNRAS, 491, 4843.

Romeo A. B., Agertz O., Renaud F., 2020, MNRAS, 499, 5656.

Schaye J., Crain R. A., Bower R. G., Furlong M., Schaller M., et al., 2015, MNRAS, 446, 521.

Schmidt M., 1959, ApJ, 129, 243

Schmidt M., 1963, ApJ, 137, 758

Schulman E., Bregman J. N., Roberts M. S., 1994, ApJ, 423, 180.

Serra P., Oosterloo T., Morganti R., Alatalo K., Blitz L., Bois M., Bournaud F., et al., 2012, MNRAS, 422, 1835.

Somerville R. S., Davé R., 2015, ARA\&A, 53, 51.

Spinelli M., Zoldan A., De Lucia G., Xie L., Viel M., 2020, MNRAS, 493, 5434.

Springel V., Hernquist L., 2003, MNRAS, 339, 289

Springel V., 2005, MNRAS, 364, 1105.

Stevens A. R. H., Lagos C. del P., Cortese L., Catinella B., Diemer B., et al., 2021, MNRAS, 502, 3158.

Stilp A. M., Dalcanton J. J., Skillman E., Warren S. R., Ott J., Koribalski B., 2013, ApJ, 773, 88.

Stiskalek R., Desmond H., Holvey T., Jones M. G., 2021, MNRAS, 506, 3205.

Tully R. B., Rizzi L., Shaya E. J., Courtois H. M., Makarov D. I., Jacobs B. A., 2009, AJ, 138, 323.

Vale A., Ostriker J. P., 2004, MNRAS, 353, 189.

Verheijen M. A. W., Sancisi R., 2001, A\&A, 370, 765.

Verheijen M. A. W., 2001, ApJ, 563, 694.

Villaescusa-Navarro F., Genel S., Castorina E., Obuljen A., Spergel D. N., et al., 2018, ApJ, 866, 135.

Vogelsberger M., Genel S., Springel V., Torrey P., Sijacki D., et al., 2014, Nature, 509, 177.

Wang J., Koribalski B. S., Serra P., van der Hulst T., Roychowdhury S., Kamphuis P., Chengalur J. N., 2016, MNRAS, 460, 2143.

Zwaan M. A., Briggs F. H., Sprayberry D., 2001, MNRAS, 327,1249

Zwaan M. A., et al., 2003, AJ, 125, 2842

Zwaan, M. A.; Meyer, M. J.; Staveley-Smith, L., 2010, MNRAS, Volume 403, Issue 4, pp. 1969-1977

\section{APPENDIX A: THE EFFECT OF $w_{\text {nr }}$ ON THE HIWF AND HIVF}

We look at the effect of varying $w_{\mathrm{nr}}$ on the derived HIVF in this section. We proceed with the approach described in section 4. We use equation 7 (Verheijen \& Sancisi 2001; Papastergis, et al. 2011) to convert $V_{\text {rot }}$ into $w_{50}$. We add $w_{\mathrm{nr}}$ to $V_{\text {rot }}$ linearly for galaxies with $V_{\text {rot }}>50 \mathrm{~km} \cdot \mathrm{s}^{-1}$ and in quadrature for galaxies with smaller velocities (Papastergis, et al. 2011). The best fit model parameters of the HIVF are 

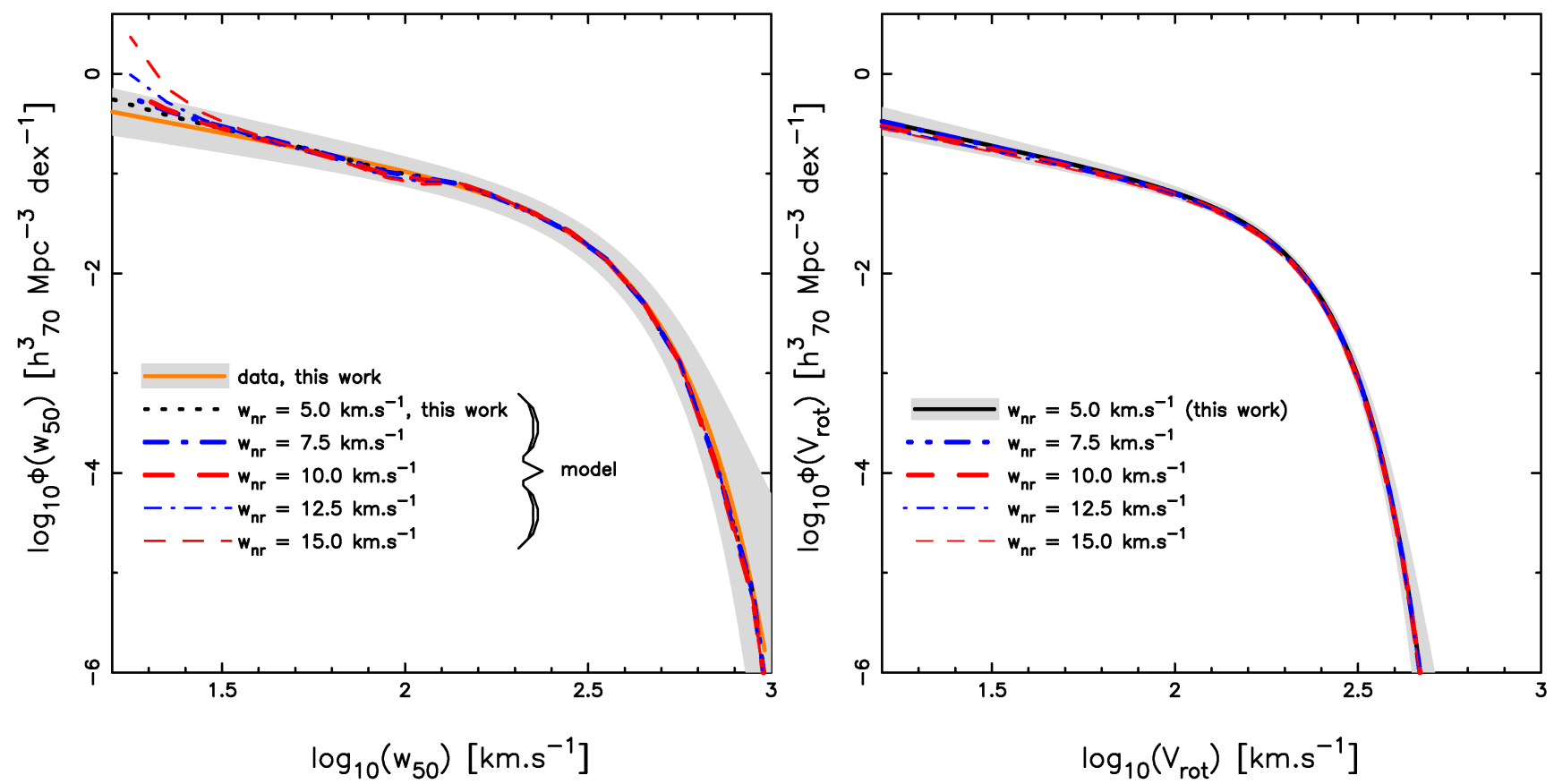

Figure A1. This figure shows the effect of varying $w_{n r}$ on the HIWF (HIVF) in the left (right) panel. In this work we considered $w_{n r}=5.0 \mathrm{~km} . \mathrm{s}^{-1}$ (see section 4). The solid line with the shaded region in the left panel represents the best fit modified Schechter function for the HIWF and its $1 \sigma$ uncertainty based on the data. The thick dotted line in the left panel represents the best fit model HIWF for $w_{n r}=$ $5.0 \mathrm{~km} . \mathrm{s}^{-1}$ which was determined by a corresponding best fit HIVF in the right panel (solid line with shaded region). The thick dot-dashed, thick dashed, thin dot-dashed, thin dashed lines represent the best fit HIWF for $w_{n r}=7.5 \mathrm{~km} . \mathrm{s}^{-1}, 10.0 \mathrm{~km} . \mathrm{s}^{-1}, 12.5 \mathrm{~km} . \mathrm{s}^{-1}, 15.0 \mathrm{~km} . \mathrm{s}^{-1}$ respectively, determined from their corresponding best fit HIVF shown on the right panel.

\begin{tabular}{lcccc}
\hline$w_{n r}$ & $\phi_{*}$ & $V_{*}$ & $\alpha$ & $\beta$ \\
\hline 5.0 & $0.0187 \pm 0.0023$ & $2.30 \pm 0.01$ & $-0.81 \pm 0.13$ & $2.70 \pm 0.17$ \\
\hline 7.5 & $0.0182 \pm 0.0023$ & $2.30 \pm 0.01$ & $-0.82 \pm 0.13$ & $2.70 \pm 0.17$ \\
\hline 10.0 & $0.0193 \pm 0.0022$ & $2.29 \pm 0.01$ & $-0.76 \pm 0.13$ & $2.64 \pm 0.17$ \\
\hline 12.5 & $0.0189 \pm 0.0022$ & $2.29 \pm 0.01$ & $-0.75 \pm 0.13$ & $2.65 \pm 0.17$ \\
\hline 15.0 & $0.0182 \pm 0.0022$ & $2.29 \pm 0.01$ & $-0.76 \pm 0.13$ & $2.65 \pm 0.17$ \\
\hline
\end{tabular}

Table A1. Best fit values with $1 \sigma$ uncertainties of modified Schechter function for HIVFs for different values of $w_{n r}$.

determined by a minimization procedure so that the corresponding model HIWF and the observed HIWF agree with each other. Our results are shown in figure A1. The right panel shows the best fit HIVF for values of $w_{\mathrm{nr}}$ in the range $5-15 \mathrm{~km} . \mathrm{s}^{-1}$ (Stilp et al. 2013). The left panel shows the corresponding model HIWF and the HIWF from observations (thick solid line with shaded region). We find negligible variation in the model HIVFs as a function of $w_{\mathrm{nr}}$. This is also true for the model HIWFs for $w_{50} \gtrsim 30 \mathrm{~km} . \mathrm{s}^{-1}$. For $w_{50}<30 \mathrm{~km} . \mathrm{s}^{-1}$ there is an upturn in the model HIWFs for $w_{\mathrm{nr}} \geq 12.5 \mathrm{~km} . \mathrm{s}^{-1}$ which is not consistent with data.

The parameters of the best fit HIVFs for different values of $w_{\mathrm{nr}}$ are shown in table A1. Although the HIVFs are consistent with each other, the effect of varying $w_{\mathrm{nr}}$ shows up most significantly at lower velocities, i.e. in the slope $\alpha$. 